\title{
Motion of Level Sets by Mean Curvature IV
}

\author{
By Lawrence C. Evans and Joel Spruck
}

\begin{abstract}
We continue our investigation of the "level-set" technique for describing the generalized evolution of hypersurfaces moving according to their mean curvature. The principal assertion of this paper is a kind of reconciliation with the geometric measure theoretic approach pioneered by $\mathrm{K}$. Brakke: we prove that almost every level set of the solution to the mean curvature evolution PDE is in fact a unit-density varifold moving according to its mean curvature. In particular, a.e. level set is endowed with a kind of "geometric structure." The proof utilizes compensated compactness methods to pass to limits in various geometric expressions.
\end{abstract}

\section{Introduction}

In several earlier papers we, Chen et al. [3,4], and others have studied a new notion of generalized mean curvature motion of hypersurfaces in $\mathbb{R}^{n}$, defined as follows. Given, say, a smooth, bounded $(n-1)$-dimensional surface $\Gamma_{0} \subset \mathbb{R}^{n}$, choose a smooth function $g: \mathbb{R}^{n} \rightarrow \mathbb{R}$ whose zero set is $\Gamma_{0}$; that is,

$$
\Gamma_{0}=\left\{x \in \mathbb{R}^{n} \mid g(x)=0\right\} .
$$

We consider next the mean curvature evolution PDE

$$
\left.\begin{array}{ll}
u_{t}=\left(\delta_{i j}-\frac{u_{x_{i}} u_{x_{j}}}{|D u|^{2}}\right) u_{x_{i} x_{j}} & \text { in } \mathbb{R}^{n} \times(0, \infty) \\
u=g & \text { in } R^{n} \times\{t=0\} .
\end{array}\right\}
$$

As explained in [8], [3], etc., this PDE asserts each level set of $u$ is evolving by mean curvature motion, at least in regions where $u$ is smooth and $|D u| \neq 0$. We and Chen et al. $[3,4]$ have shown (1.2) has a unique, weak solution $u$ (see [8], [3] for definitions), and consequently we can define $\Gamma_{t}$ to be the zero-set of $u$ at time $t$ :

$$
\Gamma_{t}=\left\{x \in \mathbb{R}^{n} \mid u(x, t)=0\right\} .
$$

Math Subject Classification Primary 58G20, 32M99. Secondary 53B35, 32M15, $22 \mathrm{E} 30$.

Key Words and Phrases Mean curvature motion, compensated compactness.

L.C.E. supported in part by NSF Grant DMS-89-03328. J.S. supported in part by NSF Grant DMS-85-01952 and DOE Grant DE-FG02-86ER25015. 
The collection of sets $\left\{\Gamma_{t}\right\}_{t \geq 0}$ comprise the (level-set method) generalized mean curvature motion, starting from the initial surface $\Gamma_{0}$. (Chen et al. [3] in fact study more general geometric laws of motion.)

The intention of this endeavor is the construction of a reasonable and useful global interpretation for mean curvature flow, which allows for developing singularities, changes of topological type, and so forth. We and other authors have consequently devoted considerable effort to studying the evolution $\Gamma_{0} \mapsto \Gamma_{t}(t>0)$, to ascertain if indeed the level-set construction above is natural geometrically. For instance, see $[8, \S 6]$ for a proof that $\left\{\Gamma_{t}\right\}_{t \geq 0}$ agrees with the smooth, classical motion by mean curvature starting from $\Gamma_{0}$, until the time $t_{*}$ of the onset of singularities in the classical motion. For later times $t \geq t_{*}$, the generalized motion $\Gamma_{t}$ is still defined, and so the real question is whether the definition (1.3) is thereafter geometrically reasonable or not.

This question is particularly pressing since others have proposed different constructions for generalized mean curvature motions. The most notable alternative approach is due to $\mathrm{K}$. Brakke, whose pioneering monograph [2] sets forth an entirely different interpretation of generalized surfaces, called varifolds, moving by curvature. Brakke's important accomplishments were first to construct a family $\left\{V_{t}\right\}_{t \geq 0}$ of evolving varifolds and second to establish partial regularity for any unit-density varifold moving via mean curvature. His methods, which build on Allard's fundamental paper [1], avoid certain of the pathologies associated with our level set approach: see $[8, \S 8]$ for a preliminary discussion.

The principal result of this current paper is a kind of reconciliation between the level-set and varifold approaches, at least generically. We will in effect prove, under certain assumptions on the initial function $g$, that almost every level set of $u$ is a unit-density varifold evolving by mean curvature in Brakke's sense. This assertion, the exact meaning of which will be set forth in Section 7, is useful on several counts. First, even though for a given initial set $\Gamma_{0}=\left\{x \in \mathbb{R}^{n} \mid g(x)=0\right\}$ the level-set flow and varifold flows may differ substantially, for "most" initial sets $\Gamma_{0}^{\gamma}=\left\{x \in \mathbb{R}^{n} \mid g(x)=\gamma\right\}$ the evolutions are compatible. Second, since the typical level set is a unit-density varifold, the partial regularity theory from Brakke [2, Chap. 6] applies. This is of interest since Brakke's own construction [2, Chap. 4] is apparently not known to yield unit-density moving varifolds. Finally, and more generally, our results demonstrate that a.e. level set of the solution of (1.2) is naturally endowed with a kind of geometric structure.

The key to our proof is a new estimate for the approximate problems introduced in [8]:

$$
\left.\begin{array}{ll}
u_{t}^{\epsilon}=\left(\delta_{i j}-\frac{u_{x_{i}}^{\epsilon} u_{x_{j}}^{\epsilon}}{\left|D u^{\epsilon}\right|^{2}+\epsilon^{2}}\right) u_{x_{i} x_{j}}^{\epsilon} & \text { in } \mathbb{R}^{n} \times(0, \infty) \\
u^{\epsilon}=g & \text { in } R^{n} \times\{t=0\} .
\end{array}\right\}
$$

In light of $[8, \S 4]$ we know $u^{\epsilon} \rightarrow u$ locally uniformly in $\mathbb{R}^{n} \times[0, \infty), u$ being the unique weak solution of (1.2). Set

$$
H^{\epsilon}=\operatorname{div}\left(\frac{D u^{\epsilon}}{\left(\left|D u^{\epsilon}\right|^{2}+\epsilon^{2}\right)^{1 / 2}}\right)
$$

a quantity which we will see can be interpreted as the approximate mean curvature of the level sets 
of $u^{\epsilon}$. Our primary new estimate is the $L^{1}$ bound

$$
\sup _{0 \leq \epsilon<1} \sup _{t \geq 0} \int_{\mathbb{R}^{n}}\left|H^{\epsilon}\right| d x<\infty
$$

proved in Section 2 . To understand the utility of this bound, let us contrast it with the easier inequality

$$
\sup _{0 \leq \epsilon<1} \int_{0}^{T} \int_{\mathbb{R}^{n}}\left|H^{\epsilon}\right|^{2}\left(\left|D u^{\epsilon}\right|^{2}+\epsilon^{2}\right)^{1 / 2} d x d t<\infty
$$

for $T>0$. Now (1.6) has a direct geometric interpretation. Indeed, let us for heuristic purposes set $\epsilon=0$ and suppose our solution $u$ of (1.2) is really smooth, with $|D u| \neq 0$. Then each level surface $\Gamma_{t}^{\gamma}=\left\{x \in \mathbb{R}^{n} \mid u(x, t)=\gamma\right\}$ moves by mean curvature, whence classical differential geometry (cf. Huisken [14]) provides the calculation

$$
\frac{d}{d t} H^{n-1}\left(\Gamma_{t}^{\gamma}\right)=-\int_{\Gamma_{t}^{\gamma}} H^{2} d H^{n-1}
$$

$H^{n-1}$ denoting $(n-1)$-dimensional Hausdorff measure and $H$ the mean curvature of $\Gamma_{t}^{\gamma}$. Note $H=\operatorname{div}(D u /|D u|)$, as $v=D u /|D u|$ is a unit normal vector field to the level set $\Gamma_{t}^{\gamma}$. Now (1.7) implies

$$
H^{n-1}\left(\Gamma_{T}^{\gamma}\right)+\int_{0}^{T} \int_{\Gamma_{t}^{\gamma}} H^{2} d H^{n-1} d t=H^{n-1}\left(\Gamma_{0}^{\gamma}\right)
$$

Integrate this equality with respect to $\gamma$ and employ the Coarea Formula to deduce

$$
\left.\int_{\mathbb{R}^{n}}|D u| d x\right|_{t=T}+\int_{0}^{T} \int_{\mathbb{R}^{n}} H^{2}|D u| d x d t=\int_{\mathbb{R}^{n}}|D g| d x .
$$

In particular,

$$
\int_{0}^{T} \int_{\mathbb{R}^{n}} H^{2}|D u| d x d t<\infty
$$

Estimate (1.5) is quite different in character, corresponding to the formal estimate on $u$ :

$$
\sup _{t>0} \int_{\mathbb{R}^{n}}|H| d x<\infty
$$

Since the integrand does not contain the term $|D u|$, we cannot invoke the Coarea Formula to prove this from the corresponding law of motion of each level set taken separately. In other words, the formal estimate (1.10) presumably depends on more than just the fact that each level set moves independently by curvature motion. Indeed, (1.10) seems somehow to be an analytic consequence of the fitting together of the level sets to foliate space. We loosely interpret this as a kind of dynamic "stability" condition on the level sets $\Gamma_{t}^{\gamma}$. In crude geometric terms, it is presumably this "stability" of the level sets that accounts for the fairly strong conclusion that generically they are unit-density varifolds 
moving by mean curvature. (For our time-dependent problem there is no obvious "minimization" principle; "stability," however ill-defined, seems to be the best we can hope for.)

The analytic consequences of (1.10), or more precisely (1.5), are interesting. As we will prove in Section 3, (1.5) implies

$$
\left|D u^{\epsilon}\right| \rightarrow|D u| \text { weakly } * \text { in } L^{\infty}\left(\mathbb{R}^{n}\right)
$$

for each time $t \geq 0$, and in particular,

$$
\int_{B}\left|D u^{\epsilon}\right| d x \rightarrow \int_{B}|D u| d x
$$

for each bounded Borel set $B$. This is a powerful statement, much stronger than the usual lower semicontinuity assertion

$$
\int_{B}|D u| d x \leq \liminf _{\epsilon \rightarrow 0} \int_{B}\left|D u^{\epsilon}\right| d x
$$

From (1.12) and the coarea formula we see in particular that "generically" the level sets of $u^{\epsilon}$ do not "lose area" as they converge to the level sets of $u$. The informal idea is that almost every level set does not "fold over" or "double up" in the limit, and so the limits are unit density.

Our proof of (1.11) from estimate (1.5) employs the method of compensated compactness, which for the case at hand amounts to a PDE/level-set version of geometric varifold techniques. Details appear in Section 3. In Sections 4 and 5 we pass to limits as $\epsilon \rightarrow 0$ in various nonlinear expressions involving $u^{\epsilon}$. We deduce for the limit $u$ level-set versions of the first variation formula for area and the inequality for motion by mean curvature. Then in Section 6 we "decompose" these formulas into corresponding statements for a.e. level set at a.e. time. Finally in Section 7 we rapidly recall the standard terminology for varifolds and moving varifolds, and we reinterpret the conclusions of Section 6 in this language. Here we very closely follow Brakke [2].

The level-set method for studying evolution of surfaces by mean-curvature flow seems to have originated in the physics literature, in the paper by Ohta et al. [17]. Sethian [19],[20] and Osher and Sethian [18] independently introduced this idea as a computational tool. Some recent theoretical analyses and applications of the level-set approach include Soner [22], Giga et al. [12], Chen et al. [4], Giga et al. [13], Ilmanen [15], and others. Ilmanen [16] has introduced a new "approximate" variational principle within the geometric measure theoretic approach and in particular has constructed a family of unit-density varifolds flowing by mean curvature. The paper by Evans et al. [7] proves the level-set generalized motion governs the asymptotic limiting behavior of the standard Allen-Cahn equation, a simple model for certain phase transitions. This provides further confirmation of the physical and geometric reasonableness of our model. 


\section{An $L^{1}$ estimate on $H^{\epsilon}$}

We first turn our attention to the approximate problem

$$
\left.\begin{array}{ll}
u_{t}^{\epsilon}=\left(\delta_{i j}-\frac{u_{x_{i}}^{\epsilon} u_{x_{j}}^{\epsilon}}{\left|D u^{\epsilon}\right|^{2}+\epsilon^{2}}\right) u_{x_{i} x_{j}}^{\epsilon} & \text { in } \mathbb{R}^{n} \times(0, \infty) \\
u^{\epsilon}=g & \text { on } \mathbb{R}^{n} \times\{t=0\}
\end{array}\right\}
$$

for $0<\epsilon \leq 1$. Here $D u^{\epsilon}=D_{x} u^{\epsilon}=\left(u_{x_{1}}^{\epsilon}, \ldots, u_{x_{n}}^{\epsilon}\right)$ is the gradient with respect to $x$. As explained in $[8, \S 4]$, this PDE has a unique, smooth solution $u^{\epsilon}$, and additionally the maximum principle provides the bounds

$$
\sup _{0<\epsilon \leq 1}\left\|u^{\epsilon}, D u^{\epsilon}, u_{t}^{\epsilon}\right\|_{L^{\infty}\left(\mathbb{R}^{n} \times(0, \infty)\right)} \leq C\|g\|_{C^{1.1}\left(\mathbb{R}^{n}\right)} .
$$

The functions $\left\{u^{\epsilon}\right\}_{\epsilon>0}$ are thus uniformly bounded and Lipschitz in $\mathbb{R}^{n} \times(0, \infty)$. In addition, we proved in $[8, \S 4.3]$ that

$$
u^{\epsilon} \rightarrow u \text { locally uniformly on } \mathbb{R}^{n} \times[0, \infty),
$$

$u$ denoting the unique weak solution of the mean curvature evolution PDE

$$
\left.\begin{array}{ll}
u_{t}=\left(\delta_{i j}-\frac{u_{x_{i}} u_{x_{j}}}{|D u|^{2}}\right) u_{x_{i} x_{j}} & \text { in } \mathbb{R}^{n} \times(0, \infty) \\
u=g & \text { on } \mathbb{R}^{n} \times\{t=0\}
\end{array}\right\}
$$

Observe in particular that (2.3) asserts the full limit exists as $\epsilon \rightarrow 0$.

Assume hereafter that

$$
\begin{aligned}
& \Gamma_{0} \text { is a smooth connected hypersurface, the } \\
& \text { boundary of a bounded open set } U \subset \mathbb{R}^{n} \text {. }
\end{aligned}
$$

We next choose the initial function $g$ to vanish on $\Gamma_{0}$ and be particularly well behaved. Recall from $[8, \S 5]$ that the evolution of any initial level set depends only on that set and not on the choice of other level sets. As we are interested in proving an $L^{1}$ bound on

$$
H^{\epsilon}=\operatorname{div}\left(\frac{D u^{\epsilon}}{\left(\left|D u^{\epsilon}\right|^{2}+\epsilon^{2}\right)^{1 / 2}}\right)
$$

we first need to show we can select the initial function $g$ so that at least at time $0, H^{\epsilon}$ is bounded in $L^{1}$. 
Lemma 2.1. There exists a $C^{3}$ function $g: \mathbb{R}^{n} \rightarrow \mathbb{R}$ such that

(i) $-1 \leq g \leq 1$,

(ii) $\Gamma_{0}=\left\{x \in \mathbb{R}^{n} \mid g(x)=0\right\}$,

(iii) $g(x)=1$ for large $|x|$,

(iv) $\sup _{0<\epsilon \leq 1} \int_{\mathbb{R}^{n}}\left|\operatorname{div}\left(\frac{D g}{\left(|D g|^{2}+\epsilon^{2}\right)^{1 / 2}}\right)\right| d x<\infty$.

Proof. 1. Let

$$
d(x)= \begin{cases}-\operatorname{dist}\left(x, \Gamma_{0}\right) & x \in \bar{U} \\ \operatorname{dist}\left(x, \Gamma_{0}\right) & x \in \mathbb{R}^{n}-\bar{U}\end{cases}
$$

denote the signed distance function to $\Gamma_{0}$. Choose $\delta_{0}$ so small $d$ is smooth within the region

$$
R=\left\{x \in \mathbb{R}^{n} \mid \operatorname{dist}\left(x, \Gamma_{0}\right)<\delta_{0}\right\}
$$

Note $|D d|=1$ and consequently

$$
d_{x_{i} x_{j}} d_{x_{i}} d_{x_{j}}=\frac{1}{2} D d \cdot D\left(|D d|^{2}\right)=0 \text { in } R .
$$

Choose a smooth function $\phi: \mathbb{R} \rightarrow \mathbb{R}$ satisfying

$$
\begin{cases}\phi(-z)=-\phi(z)(z \in \mathbb{R}), \phi(z)=-1 & \text { if } z \leq-\delta_{0} \\ \phi^{\prime}(z)>0, \phi^{\prime \prime}(z) \geq 0 & \text { if }-\delta_{0}<z \leq 0\end{cases}
$$

2. Let

$$
g(x)=\phi(d(x))\left(x \in \mathbb{R}^{n}\right)
$$

Clearly (i)-(iii) obtain, and so we must verify (iv). For this, take $x \in R$ and compute

$$
\begin{aligned}
\operatorname{div}\left(\frac{D g}{\left(|D g|^{2}+\epsilon^{2}\right)^{1 / 2}}\right) & =\frac{1}{\left(|D g|^{2}+\epsilon^{2}\right)^{3 / 2}}\left(\left(|D g|^{2}+\epsilon^{2}\right) \delta_{i j}-g_{x_{i}} g_{x_{j}}\right) g_{x_{i} x_{j}} \\
& =\frac{1}{\left(\left(\phi^{\prime}\right)^{2}+\epsilon^{2}\right)^{3 / 2}}\left(\left(\left(\phi^{\prime}\right)^{2}+\epsilon^{2}\right) \delta_{i j}-\left(\phi^{\prime}\right)^{2} d_{x_{i}} d_{x_{j}}\right)\left(\phi^{\prime} d_{x_{i} x_{j}}+\phi^{\prime \prime} d_{x_{i}} d_{x_{j}}\right) \\
& \left.=\frac{1}{\left(\left(\phi^{\prime}\right)^{2}+\epsilon^{2}\right)^{3 / 2}}\left[\left(\phi^{\prime}\right)^{2}+\epsilon^{2}\right)\left(\phi^{\prime} \Delta d+\phi^{\prime \prime}\right)-\phi^{\prime \prime}\left(\phi^{\prime}\right)^{2}\right] \text { by }(2.5) \\
& =\frac{\phi^{\prime}}{\left(\left(\phi^{\prime}\right)^{2}+\epsilon^{2}\right)^{1 / 2}} \Delta d+\frac{\epsilon^{2} \phi^{\prime \prime}}{\left(\left(\phi^{\prime}\right)^{2}+\epsilon^{2}\right)^{3 / 2}} .
\end{aligned}
$$


Consequently,

$$
\begin{aligned}
\int_{\mathbb{R}^{n}}\left|\operatorname{div}\left(\frac{D g}{\left(|D g|^{2}+\epsilon^{2}\right)^{1 / 2}}\right)\right| d x & \leq \int_{R}|\Delta d|+\frac{\epsilon^{2}\left(\phi^{\prime \prime}\right)}{\left(\left(\phi^{\prime}\right)^{2}+\epsilon^{2}\right)^{3 / 2}} d x \\
& \leq C\left(1+\epsilon^{2} \int_{-\delta_{0}}^{0} \frac{\phi^{\prime \prime}(s)}{\left(\phi^{\prime}(s)^{2}+\epsilon^{2}\right)^{3 / 2}} d s\right)
\end{aligned}
$$

where we used the Coarea Formula. But

$$
\begin{aligned}
\epsilon^{2} \int_{-\delta_{0}}^{0} \frac{\phi^{\prime \prime} d s}{\left(\left(\phi^{\prime}\right)^{2}+\epsilon^{2}\right)^{3 / 2}} & =\int_{-\delta_{0}}^{0}\left(\frac{\phi^{\prime}}{\left(\left(\phi^{\prime}\right)^{2}+\epsilon^{2}\right)^{1 / 2}}\right)^{\prime} d s \\
& =\frac{\phi^{\prime}(0)}{\left(\phi^{\prime}(0)^{2}+\epsilon^{2}\right)^{1 / 2}} \leq 1 .
\end{aligned}
$$

This calculation and (2.8) complete the proof.

Notation. We henceforth write

$$
\nu^{\epsilon}=\frac{D u^{\epsilon}}{\left(\left|D u^{\epsilon}\right|^{2}+\epsilon^{2}\right)^{1 / 2}}
$$

and

$$
H^{\epsilon}=\operatorname{div}\left(\nu^{\epsilon}\right)=\operatorname{div}\left(\frac{D u^{\epsilon}}{\left(\left|D u^{\epsilon}\right|^{2}+\epsilon^{2}\right)^{1 / 2}}\right)
$$

We think of $v^{\epsilon}$ as being the approximate normal vector field, and $H^{\epsilon}$ as being the approximate mean curvature, for the level sets of $u^{\epsilon}$. (In view of [8, §4.2], $\nu^{\epsilon}$ and $H^{\epsilon}$ are actually the normal to and mean curvature of the level sets of $v^{\epsilon} \equiv u^{\epsilon}-\epsilon x_{n+1}$ in $\mathbb{R}^{n+1}$.)

Theorem 2.2. We have the estimate

$$
\sup _{\substack{0<\in \leq 1 \\ t \geq 0}} \int_{\mathbb{R}^{n}}\left|H^{\epsilon}(x, t)\right| d x<\infty .
$$

Remark. In fact, we show

$$
t \mapsto \int_{\mathbb{R}^{n}}\left|H^{\epsilon}(x, t)\right| d x
$$

is nonincreasing. 
Proof. 1. Utilizing the notation above, we rewrite the PDE $(2.1)_{\epsilon}$ to read

$$
u_{t}^{\epsilon}=\left(\left|D u^{\epsilon}\right|^{2}+\epsilon^{2}\right)^{1 / 2} H^{\epsilon}=\left(\left|D u^{\epsilon}\right|^{2}+\epsilon^{2}\right)^{1 / 2} \operatorname{div}\left(\nu^{\epsilon}\right)
$$

2. Let us now compute

$$
v_{i, t}^{\epsilon}=\frac{u_{x_{i} t}^{\epsilon}}{\left(\left|D u^{\epsilon}\right|^{2}+\epsilon^{2}\right)^{1 / 2}}-\frac{u_{x_{i}}^{\epsilon} u_{x_{j}}^{\epsilon}}{\left(\left|D u^{\epsilon}\right|^{2}+\epsilon^{2}\right)^{3 / 2}} u_{x_{j} t}^{\epsilon}=\frac{1}{\left(\left|D u^{\epsilon}\right|^{2}+\epsilon^{2}\right)^{1 / 2}} a_{i j}^{\epsilon} u_{x_{j} t}^{\epsilon}
$$

for

$$
a_{i j}^{\epsilon} \equiv\left(\delta_{i j}-\frac{u_{x_{i}}^{\epsilon} u_{x_{j}}^{\epsilon}}{\left(\left|D u^{\epsilon}\right|^{2}+\epsilon^{2}\right)}\right) \quad(i, j=1, \ldots, n)
$$

Consequently,

$$
\begin{aligned}
H_{t}^{\epsilon} & =\left(v_{i, t}^{\epsilon}\right)_{x_{i}} \\
& =\left(\frac{1}{\left(\left|D u^{\epsilon}\right|^{2}+\epsilon^{2}\right)^{1 / 2}} a_{i j}^{\epsilon} u_{t x_{j}}^{\epsilon}\right)_{x_{i}} \\
& =\left(\frac{1}{\left(\left|D u^{\epsilon}\right|^{2}+\epsilon^{2}\right)^{1 / 2}} a_{i j}^{\epsilon}\left(\left(\left|D u^{\epsilon}\right|^{2}+\epsilon^{2}\right)^{1 / 2} H^{\epsilon}\right)_{x_{j}}\right)_{x_{i}} .
\end{aligned}
$$

4. Let $\eta: \mathbb{R} \rightarrow \mathbb{R}$ be smooth, convex, Lipschitz, with $\eta(0)=0$. Then, utilizing Lemma 2.3 below, we compute

$$
\begin{aligned}
\frac{d}{d t} \int_{\mathbb{R}^{n}} \eta\left(H^{\epsilon}\right) d x= & \int_{\mathbb{R}^{n}} \eta^{\prime}\left(H^{\epsilon}\right) H_{t}^{\epsilon} d x \\
= & \int_{\mathbb{R}^{n}} \eta^{\prime}\left(H^{\epsilon}\right)\left(\frac{1}{\left(\left|D u^{\epsilon}\right|^{2}+\epsilon^{2}\right)^{1 / 2}} a_{i j}^{\epsilon}\left(\left(\left|D u^{\epsilon}\right|^{2}+\epsilon^{2}\right)^{1 / 2} H^{\epsilon}\right)_{x_{j}}\right)_{x_{i}} d x \\
= & -\int_{\mathbb{R}^{n}} \eta^{\prime \prime}\left(H^{\epsilon}\right) \frac{H_{x_{i}}^{\epsilon}}{\left(\left|D u^{\epsilon}\right|^{2}+\epsilon^{2}\right)^{1 / 2}} a_{i j}^{\epsilon}\left(\left(\left|D u^{\epsilon}\right|^{2}+\epsilon^{2}\right)^{1 / 2} H^{\epsilon}\right)_{x_{j}} d x \\
= & -\int_{\mathbb{R}^{n}} \eta^{\prime \prime}\left(H^{\epsilon}\right) a_{i j}^{\epsilon} H_{x_{i}}^{\epsilon} H_{x_{j}}^{\epsilon} d x \\
& -\int_{\mathbb{R}^{n}} \eta^{\prime \prime}\left(H^{\epsilon}\right) \frac{H_{x_{i}}^{\epsilon}}{\left(\left|D u^{\epsilon}\right|^{2}+\epsilon^{2}\right)^{1 / 2}} a_{i j}^{\epsilon}\left(\left|D u^{\epsilon}\right|^{2}+\epsilon^{2}\right)_{x_{j}}^{1 / 2} H^{\epsilon} d x .
\end{aligned}
$$

As $\eta$ is convex and the matrix $\left(\left(a_{i j}^{\epsilon}\right)\right)$ is positive definite, the first integral in the last expression is 
nonnegative. Discarding this term and integrating in time we deduce

$$
\begin{aligned}
\int_{\mathbb{R}^{n}} \eta\left(H^{\epsilon}(x, t)\right) d x \leq & \int_{\mathbb{R}^{n}} \eta\left(H^{\epsilon}(x, 0)\right) d x \\
& +C_{\epsilon} \int_{0}^{t} \int_{\mathbb{R}^{n}} \eta^{\prime \prime}\left(H^{\epsilon}\right)\left|H^{\epsilon}\right|\left|D H^{\epsilon}\right| d x d s
\end{aligned}
$$

for $C_{\epsilon} \equiv \frac{C}{\epsilon}\left\|D\left(\left(\left|D u^{\epsilon}\right|^{2}+\epsilon^{2}\right)^{1 / 2}\right)\right\|_{L^{x}}$

5. Since we have absolutely no control over $C_{\epsilon}$ as $\epsilon \rightarrow 0$, we must select $\eta$ so that the term involving $C_{\epsilon}$ in fact vanishes. For each $\delta>0$, choose a smooth convex function $\eta_{\delta}: \mathbb{R} \rightarrow \mathbb{R}$ such that

$$
\left.\begin{array}{l}
\eta_{\delta}(0)=0, \eta_{\delta}(z) \rightarrow|z| \text { uniformly on } \mathbb{R}, \eta_{\delta} \geq 0 \\
0 \leq \eta_{\delta}^{\prime \prime} \leq \frac{c}{\delta}, \text { spt } \eta_{\delta}^{\prime \prime} \subset(-\delta, \delta)
\end{array}\right\}
$$

Set $\eta=\eta_{\delta}$ in (2.15):

$$
\int_{\mathbb{R}^{n}} \eta_{\delta}\left(H^{\epsilon}(x, t)\right) d x \leq \int_{\mathbb{R}^{n}} \eta_{\delta}\left(H^{\epsilon}(x, 0)\right) d x+\frac{C_{\epsilon}}{\delta} \int_{0}^{t} \int_{\left.|| H^{\epsilon} \mid \leq \delta\right\}}\left|H^{\epsilon}\right|\left|D H^{\epsilon}\right| d x d t .
$$

Sending $\delta \rightarrow 0$ and noting $\int_{\mathbb{R}^{n}}\left|D H^{\epsilon}\right| d x<\infty$ according to Lemma 2.3, we deduce

$$
\begin{aligned}
\int_{\mathbb{R}^{n}}\left|H^{\epsilon}(x, t)\right| d x & \leq \int_{\mathbb{R}^{n}}\left|H^{\epsilon}(x, 0)\right| d x+C_{\epsilon} \int_{0}^{t} \int_{\left\{\left|H^{\epsilon}\right|=0\right\}}\left|D H^{\epsilon}\right| d x d t \\
& =\int_{\mathbb{R}^{n}}\left|H^{\epsilon}(x, 0)\right| d x
\end{aligned}
$$

since $D H^{\epsilon}=0$ a.e. on the set $\left\{H^{\epsilon}=0\right\}$. But

$$
H^{\epsilon}(x, 0)=\operatorname{div}\left(\frac{D g}{\left(|D g|^{2}+\epsilon^{2}\right)^{1 / 2}}\right)
$$

and consequently

$$
\sup _{0<\epsilon \leq 1} \int_{\mathbb{R}^{n}}\left|H^{\epsilon}(x, 0)\right| d x<\infty
$$

according to Lemma 2.1(iv).

Lemma 2.3. For each $\epsilon>0$ there exist positive constants $A_{\epsilon}, a_{\epsilon}$ such that

$$
\left|H^{\epsilon}\right|,\left|D H^{\epsilon}\right| \leq A_{\epsilon} e^{-a_{\epsilon}|x|^{2}}
$$

for all $x \in \mathbb{R}^{n}, 0 \leq t \leq T$. 
Proof. 1. Set $w^{\epsilon}=1-u^{\epsilon}$. Then $w^{\epsilon}$ is smooth, bounded, nonnegative, and $(2.1)_{\epsilon}$ states

$$
w_{t}^{\epsilon}=a_{i j}^{\epsilon} w_{x_{i} x_{j}}^{\epsilon} \text { in } \mathbb{R}^{n} \times(0, \infty)
$$

for

$$
a_{i j}^{\epsilon} \equiv \delta_{i j}-\frac{u_{x_{i}}^{\epsilon} u_{x_{j}}^{\epsilon}}{\left(\left|D u^{\epsilon}\right|^{2}+\epsilon^{2}\right)}
$$

Since $\left|D u^{\epsilon}\right|$ is bounded, the coefficients $\left(\left(a_{i j}^{\epsilon}\right)\right)$ are uniformly elliptic. Since $u^{\epsilon}(x, 0)=g(x)=1$ for, say, $|x| \geq R$, we have

$$
w^{\epsilon}(x, 0)=0 \text { if }|x| \geq R
$$

From (2.17), (2.18) we deduce

$$
\left|w^{\epsilon}\right| \leq B_{\epsilon} e^{-b_{\epsilon}|x|^{2}}
$$

for constants $B_{\epsilon}, b_{\epsilon}>0$.

2. Write

$$
C(x, r)=B(x, r) \times[0, T] .
$$

Then since $u^{\epsilon}$ and thus the coefficients $a_{i j}^{\epsilon}$ are smooth, we deduce from (2.17), (2.18) the local bounds

$$
\left\|D^{k} w^{\epsilon}\right\|_{L^{\infty}(C(x, 1))} \leq C_{\epsilon, k}\left\|w^{\epsilon}\right\|_{L^{\infty}(C(x, 2))}
$$

for $k=1,2, \ldots$ and $|x|>R+2$. In particular, (2.18) implies

$$
\left|D^{2} u^{\epsilon}(x, t)\right|,\left|D^{3} u^{\epsilon}(x, t)\right| \leq C_{\epsilon} e^{-\epsilon_{\epsilon}|x|^{2}}
$$

for $|x|>R+2,0 \leq t \leq T$, and appropriate constants $C_{\epsilon}, c_{\epsilon}>0$. Since

$$
\left|H^{\epsilon}\right| \leq \frac{C}{\epsilon}\left|D^{2} u^{\epsilon}\right|
$$

and

$$
\left|D H^{\epsilon}\right| \leq \frac{C}{\epsilon}\left|D^{3} u^{\epsilon}\right|,
$$

estimate (2.10) follows from (2.20).

Remark. There is a somewhat more elegant technique which avoids the need for estimates on $u^{\epsilon}, H^{\epsilon}$, etc. as $|x| \rightarrow \infty$. First we fix a cube $Q$ so large that $g \equiv 1$ in $\mathbb{R}^{n}-Q$. We then redefine $g$ in $\mathbb{R}^{n}-Q$ so that $g$ is $Q$-periodic in $\mathbb{R}^{n}$. Then, by uniqueness, $u^{\epsilon}$ and thus $u$ are $Q$-periodic, 
and we can replace the integral over $\mathbb{R}^{n}$ with an integral over the finite region $Q$. The level sets of $u$ within the cube $Q$ will be unaffected.

\section{Compensated compactness; applications of $L^{1}$ estimate on $H^{\epsilon}$}

In this and subsequent sections we make use of estimate (2.11) to justify the passage to limits of various quantities as $\epsilon \rightarrow 0$. For our present purposes we may as well ignore the time variable $t$, and suppose we are given a collection $\left\{u^{\epsilon}\right\}_{0 \leq \epsilon \leq 1}$ of smooth, bounded, Lipschitz functions on $\mathbb{R}^{n}$, with the uniform estimate

$$
\sup _{0<\epsilon \leq 1}\left\|u^{\epsilon}, D u^{\epsilon}\right\|_{L^{\infty}\left(\mathbb{R}^{n}\right)}<\infty \text {. }
$$

Let us suppose also that

$$
\left.\begin{array}{ll}
u^{\epsilon} \rightarrow u & \text { locally uniformly on } \mathbb{R}^{n} \\
D u^{\epsilon} \rightarrow D u & \text { weakly } * \text { in } L^{\infty}\left(\mathbb{R}^{n} ; \mathbb{R}^{n}\right)
\end{array}\right\}
$$

as $\epsilon \rightarrow 0$, for some bounded, Lipschitz function $u$.

Also as before, we write

$$
\begin{gathered}
v^{\epsilon} \equiv \frac{D u^{\epsilon}}{\left(\left|D u^{\epsilon}\right|^{2}+\epsilon^{2}\right)^{1 / 2}}, \\
H^{\epsilon} \equiv \operatorname{div}\left(\nu^{\epsilon}\right)=\operatorname{div}\left(\frac{D u^{\epsilon}}{\left(\left|D u^{\epsilon}\right|^{2}+\epsilon^{2}\right)^{1 / 2}}\right) .
\end{gathered}
$$

We assume in addition to (3.1) that we have the estimate

$$
\sup _{0<\epsilon \leq 1}\left\|H^{\epsilon}\right\|_{L^{1}\left(\mathbb{R}^{n}\right)}<\infty .
$$

Theorem 3.1. Under the above hypotheses,

$$
\left|D u^{\epsilon}\right| \rightarrow|D u| \text { weakly } * \text { in } L^{\infty}\left(\mathbb{R}^{n}\right) .
$$

Proof. 1. We apply the machinery of compensated compactness: see, e.g., Tartar [23], Dacorogna [5], Evans [6], etc., for more details. 
First, in view of (3.2) there exists a subsequence $\epsilon_{k} \rightarrow 0$ and for a.e. $x \in \mathbb{R}^{n}$ a Borel probability measure $v_{x}$ on $\mathbb{R}^{n}$ such that

$$
F\left(D u^{\epsilon_{k}}\right) \rightarrow \bar{F} \text { weakly } * \text { in } L^{\infty}\left(\mathbb{R}^{n}\right)
$$

for each continuous function $F: \mathbb{R}^{n} \rightarrow \mathbb{R}$, where

$$
\bar{F}(x) \equiv \int_{\mathbb{R}^{n}} F(\lambda) d v_{x}(\lambda) \quad\left(\text { a.e. } x \in \mathbb{R}^{n}\right)
$$

The collection $\left\{v_{x}(\cdot)\right\}_{\text {a.e. }} \in \mathbb{R}^{n}$ comprises the Young measures associated with the weak convergence $D u^{\epsilon_{k}} \rightarrow D u$.

2. Since $\left|v^{\epsilon}\right| \leq 1$, passing to a further subsequence as necessary and reindexing, we may assume

$$
v^{\epsilon_{k}} \rightarrow v \text { weakly } * \text { in } L^{\infty}\left(\mathbb{R}^{n} ; \mathbb{R}^{n}\right)
$$

for

$$
|v| \leq 1 \text { in } \mathbb{R}^{n}
$$

Utilizing estimate (3.5) we may additionally suppose that there exists a signed Radon measure $\mu$ on $\mathbb{R}^{n}$ such that

$$
H^{\epsilon_{k}} \rightarrow \mu \text { weakly as measures on } \mathbb{R}^{n}
$$

Thus

$$
\int_{\mathbb{R}^{n}} w H^{\epsilon_{k}} d x \rightarrow \int_{\mathbb{R}^{n}} w d \mu
$$

for each continuous function $w$ with compact support. If, in particular, $w$ is Lipschitz and has compact support, we have

$$
\begin{aligned}
\int_{\mathbb{R}^{n}} w d \mu & =\lim \int_{\mathbb{R}^{n}} w H^{\epsilon_{k}} d x \\
& =-\lim _{k \rightarrow \infty} \int_{\mathbb{R}^{n}} D w \cdot v^{\epsilon_{k}} d x \\
& =-\int_{\mathbb{R}^{n}} D w \cdot v d x .
\end{aligned}
$$

3. Now we claim

$$
D u^{\epsilon_{k}} \cdot v^{\epsilon_{k}} \rightarrow D u \cdot v \text { weakly } * \text { in } L^{\infty}\left(\mathbb{R}^{n}\right)
$$


This is a variant of the Div-Curl Lemma (cf. [23],[5], etc.). Indeed, letting $\phi \in C_{c}^{\infty}\left(\mathbb{R}^{n}\right)$ we discover

$$
\begin{aligned}
\lim _{k \rightarrow \infty} \int_{\mathbb{R}^{n}} D u^{\epsilon_{k}} \cdot v^{\epsilon_{k}} \phi d x & =-\lim _{k \rightarrow \infty} \int_{\mathbb{R}^{n}} u^{\epsilon_{k}} H^{\epsilon_{k}} \phi+u^{\epsilon_{k}} \nu^{\epsilon_{k}} \cdot D \phi d x \\
& =-\int_{\mathbb{R}^{n}} u \phi d \mu-\int_{\mathbb{R}^{n}} u v \cdot D \phi d x \\
& =\int_{\mathbb{R}^{n}} D(u \phi) \cdot v d x-\int_{\mathbb{R}^{n}} u v \cdot D \phi d x \text { by (3.12) } \\
& =\int_{\mathbb{R}^{n}} D u \cdot v \phi d x
\end{aligned}
$$

Hence $D u^{\epsilon_{k}} \cdot v^{\epsilon_{k}}$ converges to $D u \cdot v$ in the distribution sense. But $\left\{D u^{\epsilon_{k}} \cdot v^{\epsilon_{k}}\right\}_{k=1}^{\infty}$ is bounded in $L^{\infty}\left(\mathbb{R}^{n}\right)$ and so converges weakly $*$ in $L^{\infty}\left(\mathbb{R}^{n}\right)$ as well. This verifies claim (3.13).

4. Next note the elementary inequality

$$
\begin{aligned}
|| p\left|-\frac{|p|^{2}}{\left(|p|^{2}+\epsilon^{2}\right)^{1 / 2}}\right| & =\frac{|p|}{\left(|p|^{2}+\epsilon^{2}\right)^{1 / 2}}\left|\left(|p|^{2}+\epsilon^{2}\right)^{1 / 2}-\right| p|| \\
& \leq \epsilon \text { for all } p \in \mathbb{R}^{n} .
\end{aligned}
$$

Thus

$$
\begin{aligned}
|| D u^{\epsilon}\left|-D u^{\epsilon} \cdot v^{\epsilon}\right| & =|| D u^{\epsilon}\left|-\frac{\left|D u^{\epsilon}\right|^{2}}{\left(\left|D u^{\epsilon}\right|^{2}+\epsilon^{2}\right)^{1 / 2}}\right| \\
& \leq \epsilon \text { for all } x \in \mathbb{R}^{n}
\end{aligned}
$$

Consequently (3.13) implies

$$
\left|D u^{\epsilon_{k}}\right| \rightarrow D u \cdot v \text { weakly } * \text { in } L^{\infty}\left(\mathbb{R}^{n}\right)
$$

5. On the other hand, according to (3.7) and (3.8),

$$
\left|D u^{\epsilon_{k}}\right| \rightarrow \int_{\mathbb{R}^{n}}|\lambda| d v_{x}(\lambda)
$$

and

$$
D u=\int_{\mathbb{R}^{n}} \lambda d v_{x}(\lambda)
$$

for a.e. $x \in \mathbb{R}^{n}$. Therefore (3.14) implies

$$
\int_{\mathbb{R}^{n}}|\lambda| d v_{x}(\lambda)=\int_{\mathbb{R}^{n}} \lambda d v_{x}(\lambda) \cdot v(x)
$$


for a.e. $x \in \mathbb{R}^{n}$. But $|v(x)| \leq 1$ and so

$$
\begin{aligned}
\int_{\mathbb{R}^{n}}|\lambda| d \nu_{x}(\lambda) & \leq\left|\int_{\mathbb{R}^{n}} \lambda d v_{x}(\lambda)\right||v(x)| \\
& \leq\left|\int_{\mathbb{R}^{n}} \lambda d v_{x}(\lambda)\right| \\
& \leq \int_{\mathbb{R}^{n}}|\lambda| d v_{x}(\lambda) .
\end{aligned}
$$

Thus

$$
\begin{aligned}
\int_{\mathbb{R}^{n}}|\lambda| d \nu_{x}(\lambda) & =\left|\int_{\mathbb{R}^{n}} \lambda d v_{x}(\lambda)\right| \quad\left(\text { a.e. } x \in \mathbb{R}^{n}\right) \\
& =|D u(x)| .
\end{aligned}
$$

Hence (3.15) in fact says

$$
\left|D u^{\epsilon_{k}}\right| \rightarrow|D u| \text { weakly } * \text { in } L^{\infty}\left(\mathbb{R}^{n}\right)
$$

This assertion is valid for each subsequence $\epsilon_{k} \rightarrow \infty$, and so (3.6) is proved.

Remark 1. Note very carefully Theorem 3.1 does not say $D u^{\epsilon} \rightarrow D u$ a.e. or in $L_{\text {loc }}^{p}\left(\mathbb{R}^{n}\right)$ for any $1 \leq p \leq \infty$. Such strong convergence may in fact fail. Consider as an example:

$$
u^{\epsilon}(x)=x_{n}+\epsilon g\left(x_{n} / \epsilon\right) \quad\left(x \in \mathbb{R}^{n}, 0<\epsilon \leq 1\right)
$$

where $g: \mathbb{R} \rightarrow \mathbb{R}$ is smooth, 1-periodic, $\left|g^{\prime}\right| \leq \alpha<1$, and $\int_{0}^{1} g(t) d t=0, \int_{0}^{1}\left|g^{\prime}(t)\right| d t \neq 0$. Then $u^{\epsilon} \rightarrow u=x_{n}$ and $D u^{\epsilon}=\left(0, \ldots, 1+g^{\prime}\left(x_{n} / \epsilon\right)\right)$. Since $0<1-\alpha \leq 1+g^{\prime}\left(x_{n} / \epsilon\right) \leq 1+\alpha$, $\operatorname{div}\left(D u^{\epsilon} /\left|D u^{\epsilon}\right|\right)=0$. Thus

$$
\begin{aligned}
H^{\epsilon} & =\operatorname{div}\left(\frac{D u^{\epsilon}}{\left(\left|D u^{\epsilon}\right|^{2}+\epsilon^{2}\right)^{1 / 2}}-\frac{D u^{\epsilon}}{\left|D u^{\epsilon}\right|}\right) \\
& =\operatorname{div}\left[\frac{\left.\left(\left|D u^{\epsilon}\right|-\left(\left|D u^{\epsilon}\right|^{2}+\epsilon^{2}\right)^{1 / 2}\right) D u^{\epsilon}\right)}{\left|D u^{\epsilon}\right|\left(\left|D u^{\epsilon}\right|^{2}+\epsilon^{2}\right)^{1 / 2}}\right] .
\end{aligned}
$$

Since $0<1-\alpha \leq\left|D u^{\epsilon}\right| \leq 1+\alpha$ and $\left|D^{2} u^{\epsilon}\right| \leq C / \epsilon$, we compute

$$
\sup _{0<\epsilon \leq 1}\left|H^{\epsilon}\right| \leq O(\epsilon)\left\|D^{2} u^{\epsilon}\right\|_{L^{\infty}}=O(1)<\infty .
$$

In particular $\left\{\left|H^{\epsilon}\right|\right\}_{0<\epsilon \leq 1}$ is bounded in $L_{\text {loc }}^{\infty}\left(\mathbb{R}^{n}\right)$. But

$$
D u^{\epsilon} \nrightarrow D u=(0, \ldots, 1)
$$

a.e. or in $L_{\text {loc }}^{p}\left(\mathbb{R}^{n} ; \mathbb{R}^{n}\right)$. 
Remark 2. If $p, q \in \mathbb{R}^{n}, p, q \neq 0$, we have the identity

$$
|p|=|q|+\frac{q}{|q|} \cdot(p-q)+\frac{|p|}{2}\left|\frac{p}{|p|}-\frac{q}{|q|}\right|^{2}
$$

Assume $\bar{\lambda}=D u(x) \neq 0$. Setting $p=\lambda, q=\bar{\lambda} \neq 0$ and integrating with respect to $v_{x}(\lambda)$ over $\mathbb{R}^{n}$, we deduce

$$
\int_{\mathbb{R}^{n}}|\lambda| d v_{x}(\lambda)=|\bar{\lambda}|+\int_{\mathbb{R}^{n}} \frac{|\lambda|}{2}\left|\frac{\lambda}{|\lambda|}-\frac{\bar{\lambda}}{|\bar{\lambda}|}\right|^{2} d v_{x}(\lambda)
$$

Thus (3.18) implies

$$
\frac{|\lambda|}{2}\left|\frac{\lambda}{|\lambda|}-\frac{\bar{\lambda}}{|\bar{\lambda}|}\right|^{2}=0 \quad v_{x} \text { a.e., }
$$

and so $v_{x}$ is supported in the ray $\{t \bar{\lambda} \mid t \geq 0\} \subset \mathbb{R}^{n}$. We see from the example in Remark 1 that $v_{x}$ is however not necessarily a unit mass.

Remark 3. It is perhaps of interest to compare these deductions with those resulting from different estimates on $H^{\epsilon}$. Assume for instance that

$$
\sup _{0<\epsilon \leq 1}\left|H^{\epsilon}\right|\left(\left|D u^{\epsilon}\right|^{2}+\epsilon^{2}\right)^{1 / 2}<\infty
$$

an estimate available in the full time-dependent problem, since $\sup _{0<\epsilon \leq 1}\left|H^{\epsilon}\right|\left(\left|D u^{\epsilon}\right|^{2}+\epsilon^{2}\right)^{1 / 2}=$ $\sup _{0<\epsilon \leq 1}\left|u_{t}^{\epsilon}\right|<\infty$. Consider the expressions

$$
\Phi_{i}^{\epsilon} \equiv u_{x_{j}}^{\epsilon}\left(\left(\left|D u^{\epsilon}\right|^{2}+\epsilon^{2}\right)^{1 / 2} \delta_{i j}-\frac{u_{x_{i}}^{\epsilon} u_{x_{j}}^{\epsilon}}{\left(\left|D u^{\epsilon}\right|^{2}+\epsilon^{2}\right)^{1 / 2}}\right) \quad(i=1, \ldots, n)
$$

Now

$$
\left(\left(\left|D u^{\epsilon}\right|^{2}+\epsilon^{2}\right)^{1 / 2} \delta_{i j}-\frac{u_{x_{i}}^{\epsilon} u_{x_{j}}^{\epsilon}}{\left(\left|D u^{\epsilon}\right|^{2}+\epsilon^{2}\right)^{1 / 2}}\right)_{x_{j}}=-u_{x_{i}}^{\epsilon} H^{\epsilon}
$$

is bounded uniformly in $\epsilon$, and so the Div-Curl Lemma implies for a.e. $x$

$$
\Phi_{i}^{\epsilon} \rightarrow \bar{\lambda}_{j}\left(\int_{\mathbb{R}^{n}}|\lambda| \delta_{i j}-\frac{\lambda_{i} \lambda_{j}}{|\lambda|} d v_{x}(\lambda)\right)
$$


But we also note

$$
\begin{aligned}
\Phi_{i}^{\epsilon} & =\left[\left(\left|D u^{\epsilon}\right|^{2}+\epsilon^{2}\right)^{1 / 2}-\frac{\left|D u^{\epsilon}\right|^{2}}{\left(\left|D u^{\epsilon}\right|^{2}+\epsilon^{2}\right)^{1 / 2}}\right] u_{x_{i}}^{\epsilon} \\
& =\frac{\epsilon^{2}}{\left(\left|D u^{\epsilon}\right|^{2}+\epsilon^{2}\right)^{1 / 2}} u_{x_{i}}^{\epsilon} \rightarrow 0 .
\end{aligned}
$$

Thus

$$
\bar{\lambda}_{j}\left(\int_{\mathbb{R}^{n}}|\lambda| \delta_{i j}-\frac{\lambda_{i} \lambda_{j}}{|\lambda|} d \nu_{x}(\lambda)\right)=0 \quad(i=1, \ldots, n)
$$

Multiply by $\bar{\lambda}_{i}$ and sum on $i$ :

$$
\int_{\mathbb{R}^{n}}|\lambda||\bar{\lambda}|^{2}-\frac{(\lambda \cdot \bar{\lambda})^{2}}{|\lambda|} d v_{x}(\lambda)=0
$$

The integrand is nonnegative, and thus

$$
|\lambda|^{2}|\bar{\lambda}|^{2}=(\lambda \cdot \bar{\lambda})^{2} \quad v_{x} \text { a.e. }
$$

Hence Young's measure $v_{x}$ is supported in the line $\{t \bar{\lambda} \mid t \in \mathbb{R}\}$, but we cannot thereby conclude (as in Remark 2) that $v_{x}$ is supported in the ray $\{t \bar{\lambda} \mid t \geq 0\}$. This stronger result seems to require an estimate on $H^{\epsilon}$ like (2.11), which does not involve the term $\left|D u^{\epsilon}\right|$.

Our goal next is to characterize $\nu$, the weak $*$ limit of the $\left\{\nu^{\epsilon_{k}}\right\}_{k=1}^{\infty}$. Observe that since the mapping $p \mapsto p /|p|$ is not continuous, we cannot employ Young measures to write $v(x)=$ $\int_{\mathbb{R}^{n}}(\lambda /|\lambda|) d \nu_{x}(\lambda)$ for a.e. $x \in \mathbb{R}^{n}$. Nevertheless, we can show;

Theorem 3.2. Assume

$$
v^{\epsilon_{k}} \rightarrow v \text { weakly } * \text { in } L^{\infty}\left(\mathbb{R}^{n} ; \mathbb{R}^{n}\right)
$$

Then

$$
v=\frac{D u}{|D u|} \text { a.e. on the set }\{|D u|>0\}
$$

Proof. 1. We extract more information from the compensated compactness methods employed in the proof of Theorem 3.1. First recall the equality (3.18), which asserts

$$
\int_{\mathbb{R}^{n}}|\lambda| d v_{x}(\lambda)=\left|\int_{\mathbb{R}^{n}} \lambda d v_{x}(\lambda)\right|
$$


for a.e. $x \in \mathbb{R}^{n},\left\{v_{x}(\cdot)\right\}$ denoting as before the Young measures corresponding to the weak convergence $D u^{\epsilon_{\Lambda}} \rightarrow D u$.

Fix $x \in \mathbb{R}^{n}$ for which (3.21) obtains and suppose

$$
D u(x)=\int_{\mathbb{R}^{n}} \lambda d v_{x}(\lambda) \equiv \bar{\lambda} \neq 0
$$

According to (3.16) in the previous proof

$$
\int_{\mathbb{R}^{n}}|\lambda| d v_{x}(\lambda)=\bar{\lambda} \cdot v(x)
$$

But

$$
\bar{\lambda} \cdot v(x) \leq|\bar{\lambda} \cdot v(x)| \leq|\bar{\lambda}||v(x)| \leq|\bar{\lambda}| \leq \int_{\mathbb{R}^{n}}|\lambda| d v_{x}
$$

and so we would obtain a contradiction to (3.23) were any of the inequalities in (3.24) strict. Thus $\nu(x)$ is a unit vector, pointing in the same direction as $\bar{\lambda}$ :

$$
v(x)=\frac{\lambda}{|\bar{\lambda}|}=\frac{D u(x)}{|D u(x)|}
$$

This conclusion is valid for a.e. $x \in\{|D u|>0\}$.

Now we show $v^{\epsilon} \rightarrow v=D u /|D u|$ strongly on the set $\{|D u|>0\}$.

Theorem 3.3. We have

$$
\frac{D u^{\epsilon}}{\left(\left|D u^{\epsilon}\right|^{2}+\epsilon^{2}\right)^{1 / 2}} \rightarrow \frac{D u}{|D u|} \text { strongly in } L_{\text {loc }}^{2}\left(\{|D u|>0\} ; \mathbb{R}^{n}\right)
$$

Proof. According to Theorem 3.2,

$$
v^{\epsilon_{\ell}} \rightarrow \frac{D u}{|D u|} \text { weakly } * \text { in }\{|D u|>0\}
$$

whenever $\left\{\nu^{\epsilon_{k}}\right\}_{k=1}^{\infty}$ is a weakly $*$ convergent subsequence. In particular, the full limit

$$
\nu^{\epsilon} \rightarrow \frac{D u}{|D u|} \text { weakly } * \text { in }\{|D u|>0\}
$$


exists. But if $\phi \in C_{c}^{\infty}\left(\mathbb{R}^{n}\right), \phi \geq 0$, and we write $v \equiv \frac{D u}{|D u|}$ on $\{|D u|>0\}$, then

$$
\begin{aligned}
\int_{\{|D u|>0\}} \phi\left|v^{\epsilon}-v\right|^{2} d x & =\int_{\{|D u|>0\}} \phi\left(\left|v^{\epsilon}\right|^{2}+|v|^{2}-2 v^{\epsilon} \cdot v\right) d x \\
& \leq 2 \int_{\{|D u|>0\}} \phi\left(1-v^{\epsilon} \cdot v\right) d x
\end{aligned}
$$

and the term on the right converges to

$$
2 \int_{\{|D u|>0\}} \phi\left(1-|v|^{2}\right) d x=0
$$

since $|v|=1$. Thus $v^{\epsilon} \rightarrow v=D u /|D u|$ strongly in $L_{\text {loc }}^{2}\left(\{|D u|>0\} ; \mathbb{R}^{n}\right)$.

Remark. As $\left|v^{\epsilon}\right|,|v| \leq 1$, the convergence above is in $L_{\text {loc }}^{p}$ as well, for $1 \leq p<\infty$.

\section{Passage to limits, I}

We return now to the full time-dependent problems $(2.1)_{\epsilon},(2.4)$. In view of estimates (2.2), (2.11) and Theorems 3.1 and 3.3, we have

$$
\left.\begin{array}{l}
\left|D u^{\epsilon}(\cdot, t)\right| \rightarrow|D u(\cdot, t)| \text { weakly } * \text { in } L^{\infty}(\mathbb{R} n) \\
\text { for each time } t \geq 0
\end{array}\right\}
$$

and

$$
\left.\begin{array}{rl}
\frac{D u^{\epsilon}(\cdot, t)}{\left(\left|D u^{\epsilon}(\cdot, t)\right|^{2}+\epsilon^{2}\right)^{1 / 2}} & \rightarrow \frac{D u(\cdot, t)}{|D u(\cdot, t)|} \text { strongly in } L_{\text {loc }}^{2}\left(\{|D u(\cdot, t)|>0\} ; \mathbb{R}^{n}\right) \\
\text { for each time } t & \geq 0 .
\end{array}\right\}
$$

Integrating in time, we deduce as well that

$$
\left|D u^{\epsilon}\right| \rightarrow|D u| \text { weakly } * \text { in } L^{\infty}\left(\mathbb{R}^{n} \times(0, \infty)\right)
$$

and

$$
\frac{D u^{\epsilon}}{\left(\left|D u^{\epsilon}\right|^{2}+\epsilon^{2}\right)^{1 / 2}} \rightarrow \frac{D u}{|D u|} \text { strongly in } L_{\mathrm{loc}}^{2}\left(\{|D u|>0\} ; \mathbb{R}^{n}\right)
$$

Our intention is to pass to limits in various geometric formulas involving $H^{\epsilon}, \frac{D u^{\epsilon}}{\left(\left|D u^{\epsilon}\right|^{2}+\epsilon^{2}\right)^{1 / 2}}$, etc., and for this we will need an additional $L^{2}$ estimate on $H^{\epsilon}$. 
Theorem 4.1. For each $T>0$ and each compact set $K \subset \mathbb{R}^{n}$,

$$
\sup _{0<\epsilon \leq 1} \int_{0}^{T} \int_{K}\left(H^{\epsilon}\right)^{2}\left(\left|D u^{\epsilon}\right|^{2}+\epsilon^{2}\right)^{1 / 2} d x d t<\infty .
$$

Proof. Select $\phi \in C_{c}^{\infty}\left(\mathbb{R}^{n}\right), 0 \leq \phi \leq 1$. Then

$$
\begin{aligned}
\frac{d}{d t} \int_{\mathbb{R}^{n}} \phi^{2}\left(\left|D u^{\epsilon}\right|^{2}+\epsilon^{2}\right)^{1 / 2} d x= & \int_{\mathbb{R}^{n}} \phi^{2} \frac{D u^{\epsilon} \cdot D u_{t}^{\epsilon}}{\left(\left|D u^{\epsilon}\right|^{2}+\epsilon^{2}\right)^{1 / 2}} d x \\
= & -\int_{\mathbb{R}^{n}} \phi^{2} \operatorname{div}\left(\frac{D u^{\epsilon}}{\left(\left|D u^{\epsilon}\right|^{2}+\epsilon^{2}\right)^{1 / 2}}\right) u_{t}^{\epsilon} d x \\
& -2 \int_{\mathbb{R}^{n}} \phi \frac{D \phi \cdot D u^{\epsilon}}{\left(\left|D u^{\epsilon}\right|^{2}+\epsilon^{2}\right)^{1 / 2}} u_{t}^{\epsilon} d x \\
\leq & -\int_{\mathbb{R}^{n}} \phi^{2}\left(H^{\epsilon}\right)^{2}\left(\left|D u^{\epsilon}\right|^{2}+\epsilon^{2}\right)^{1 / 2} d x \\
& +2 \int_{\mathbb{R}^{n}} \phi|D \phi|\left|H^{\epsilon}\right|\left(\left|D u^{\epsilon}\right|^{2}+\epsilon^{2}\right)^{1 / 2} d x \text { by (2.12) } \\
\leq & -\frac{1}{2} \int_{\mathbb{R}^{n}} \phi^{2}\left(H^{\epsilon}\right)^{2}\left(\left|D u^{\epsilon}\right|^{2}+\epsilon^{2}\right)^{1 / 2} d x \\
& +2 \int_{\mathbb{R}^{n}}|D \phi|^{2}\left(\left|D u^{\epsilon}\right|^{2}+\epsilon^{2}\right)^{1 / 2} d x .
\end{aligned}
$$

Integrating, we deduce

$$
\begin{aligned}
\int_{0}^{T} \int_{\mathbb{R}^{\prime \prime}} \phi^{2}\left(H^{\epsilon}\right)^{2}\left(\left|D u^{\epsilon}\right|^{2}+\epsilon^{2}\right)^{1 / 2} d x & \\
& \leq 4 \int_{0}^{T} \int_{\mathbb{R}^{n}}|D \phi|^{2}\left(\left|D u^{\epsilon}\right|^{2}+\epsilon^{2}\right)^{1 / 2} d x+2 \int_{\mathbb{R}^{n}} \phi^{2}\left(|D g|^{2}+\epsilon^{2}\right)^{1 / 2} d x
\end{aligned}
$$

Given a compact set $K \subset \mathbb{R}^{n}$, we choose $\phi \equiv 1$ on $K$ and then recall estimate (2.2) to complete the proof.

Consider next the quantities

$$
\left\{H^{\epsilon}\left(\left|D u^{\epsilon}\right|^{2}+\epsilon^{2}\right)^{1 / 2}\right\}_{0<\epsilon \leq 1} .
$$

According to the PDE $(2.1)_{\epsilon}$,

$$
u_{t}^{\epsilon}=\left(\left|D u^{\epsilon}\right|^{2}+\epsilon^{2}\right)^{1 / 2} H^{\epsilon}
$$

and so employing estimate (2.2) we deduce

$$
H^{\epsilon}\left(\left|D u^{\epsilon}\right|^{2}+\epsilon^{2}\right)^{1 / 2} \rightarrow u_{t} \text { weakly } * \text { in } L^{\infty}\left(\mathbb{R}^{n} \times(0, \infty)\right) .
$$


Lemma 4.2. $u_{t}=0$ a.e. on $\{|D u|=0\}$.

Proof. Let $A$ be any bounded, measurable subset of $\{|D u|=0\} \subset \mathbb{R}^{n} \times(0, \infty)$. Then

$$
\begin{aligned}
\left|\int_{A} u_{i} d x d t\right|= & \left|\lim _{\epsilon \rightarrow \infty} \int_{A} H^{\epsilon}\left(\left|D u^{\epsilon}\right|^{2}+\epsilon^{2}\right)^{1 / 2} d x d t\right| \\
\leq & \limsup _{\epsilon \rightarrow 0}\left(\int_{A}\left(H^{\epsilon}\right)^{2}\left(\left|D u^{\epsilon}\right|^{2}+\epsilon^{2}\right)^{1 / 2} d x d t\right)^{1 / 2} \\
& \times\left(\int_{A}\left(\left|D u^{\epsilon}\right|^{2}+\epsilon^{2}\right)^{1 / 2} d x d t\right)^{1 / 2} \\
\leq & C \limsup _{\epsilon \rightarrow 0}\left(\int_{A}\left(\left|D u^{\epsilon}\right|^{2}+\epsilon^{2}\right)^{1 / 2} d x d t\right)^{1 / 2},
\end{aligned}
$$

the last inequality valid owing to (4.5). Now

$$
\left|\left(\left|D u^{\epsilon}\right|^{2}+\epsilon^{2}\right)^{1 / 2}-\right| D u^{\epsilon}|| \leq \epsilon,
$$

and so

$$
\begin{aligned}
\left|\int_{A} u_{t} d x d t\right| & \leq C \limsup _{\epsilon \rightarrow 0}\left(\int_{A}\left(\left|D u^{\epsilon}\right| d x d t\right)^{1 / 2}\right. \\
& =C\left(\int_{A}|D u| d x d t\right)^{1 / 2} \text { by }(4.3) \\
& =0
\end{aligned}
$$

Next define

$$
H \equiv \begin{cases}u_{t} /|D u| & \text { if }|D u|>0 \\ 0 & \text { if }|D u|=0\end{cases}
$$

We reinterpret (4.7) and Lemma 3.2 to read

$$
H^{\epsilon}\left(\left|D u^{\epsilon}\right|^{2}+\epsilon^{2}\right)^{1 / 2}-H|D u| \text { weakly } * \text { in } L^{\infty}\left(\mathbb{R}^{n} \times(0, \infty)\right)
$$

and

$$
H|D u|=0 \text { a.e. on }\{|D u|=0\} .
$$

In addition,

$$
H|D u| \text { is essentially bounded in } \mathbb{R}^{n} \times(0, \infty)
$$


We also have an $L^{2}$ bound on $H$ :

Lemma 4.3. For each $T>0$ and each compact set $K \subset \mathbb{R}^{n}$,

$$
\int_{0}^{T} \int_{K} H^{2}|D u| d x d t<\infty
$$

Proof. Set

$$
\mu(A)=\int_{A}|D u| d x d t
$$

for Borel sets $B \subset \mathbb{R}^{n} \times(0, \infty)$, and suppose $\phi \in L^{\infty}(K \times(0, T))$. Then

$$
\begin{aligned}
\left|\int_{0}^{T} \int_{K} \phi H\right| D u|d x d t|= & \lim _{\epsilon \rightarrow 0}\left|\int_{0}^{T} \int_{K} \phi H^{\epsilon}\left(|D u|^{2}+\epsilon^{2}\right)^{1 / 2} d x d t\right| \\
\leq & \limsup _{\epsilon \rightarrow 0}\left(\int_{0}^{T} \int_{K}\left(H^{\epsilon}\right)^{2}\left(\left|D u^{\epsilon}\right|^{2}+\epsilon^{2}\right)^{1 / 2} d x d t\right) \\
& \left(\int_{0}^{T} \int_{K} \phi^{2}\left(\left|D u^{\epsilon}\right|^{2}+\epsilon^{2}\right)^{1 / 2} d x d t\right)^{1 / 2} \\
\leq & C\left(\int_{0}^{T} \int_{K} \phi^{2} d \mu\right)^{1 / 2},
\end{aligned}
$$

as in the proof of Lemma 4.2. Hence

$$
\left|\int_{0}^{T} \int_{K} \phi H d \mu\right| \leq C\left(\int_{0}^{T} \int_{K} \phi^{2} d \mu\right)^{1 / 2}
$$

for each $\phi \in L^{\infty}(K \times(0, T))$. By approximation, the same inequality obtains if $\phi \in L^{2}(K \times$ $(0, T), \mu)$, whence

$$
\int_{0}^{T} \int_{K} H^{2} d \mu=\int_{0}^{T} \int_{K} H^{2}|D u| d x d t<\infty
$$

\section{Passage to limits, II}

Next we employ the terminology and convergence assertions from Section 4 to deduce for $u$ "level-set versions" of (a) the first variation formula for area and (b) the inequality for motion by mean curvature. The idea is to interpret the function $H$ introduced in Section 4 as mean curvature and the vector $-H(D u /|D u|)$ as the mean curvature vector, at least on the set $\{|D u|>0\}$. 
Theorem 5.1. For a.e. $t \geq 0$, we have the identity

$$
\int_{\mathbb{R}^{n}}\left(\delta_{i j}-\frac{u_{x_{i}} u_{x_{j}}}{|D u|^{2}}\right) g_{x_{j}}^{i}|D u| d x=\int_{\mathbb{R}^{n}} g^{i} \frac{u_{x_{i}}}{|D u|} H|D u| d x
$$

for each vector field $g \in C_{c}^{1}\left(\mathbb{R}^{n} ; \mathbb{R}^{n}\right), g=\left(g^{1}, \ldots, g^{n}\right)$. (Each integrand is interpreted as being zero on the set $\{|D u|=0\}$.)

Proof. 1. Given $g=\left(g^{1}, \ldots, g^{n}\right)$ and $0<\epsilon \leq 1$, we compute

$$
\begin{aligned}
& \int_{\mathbb{R}^{n}} g^{i} \frac{u_{x_{i}}^{\epsilon}}{\left(\left|D u^{\epsilon}\right|^{2}+\epsilon^{2}\right)^{1 / 2}} H^{\epsilon}\left(\left|D u^{\epsilon}\right|^{2}+\epsilon^{2}\right)^{1 / 2} d x \\
& =\int_{\mathbb{R}^{n}} g^{i} u_{x_{i}}^{\epsilon}\left(\frac{u_{x_{j}}^{\epsilon}}{\left(\left|D u^{\epsilon}\right|^{2}+\epsilon^{2}\right)^{1 / 2}}\right)_{x_{j}} d x \\
& =-\int_{\mathbb{R}^{n}} g_{x_{j}}^{i} u_{x_{i}}^{\epsilon} \frac{u_{x_{j}}^{\epsilon}}{\left(\left|D u^{\epsilon}\right|^{2}+\epsilon^{2}\right)^{1 / 2}} d x-\int_{\mathbb{R}^{n}} g^{i} u_{x_{i} x_{j}}^{\epsilon} \frac{u_{x_{j}}^{\epsilon}}{\left(\left|D u^{\epsilon}\right|^{2}+\epsilon^{2}\right)^{1 / 2}} d x \\
& =-\int_{\mathbb{R}^{n}} g_{x_{j}}^{i} \frac{u_{x_{i}}^{\epsilon} u_{x_{j}}^{\epsilon}}{\left(\left|D u^{\epsilon}\right|^{2}+\epsilon^{2}\right)^{1 / 2}} d x+\int_{\mathbb{R}^{n}} g_{x_{i}}^{i}\left(\left|D u^{\epsilon}\right|^{2}+\epsilon^{2}\right)^{1 / 2} d x \\
& =\int_{\mathbb{R}^{n}}\left(\delta_{i j}-\frac{u_{x_{i}}^{\epsilon} u_{x_{j}}^{\epsilon}}{\left(\left|D u^{\epsilon}\right|^{2}+\epsilon^{2}\right)}\right) g_{x_{j}}^{i}\left(\left|D u^{\epsilon}\right|^{2}+\epsilon^{2}\right)^{1 / 2} d x .
\end{aligned}
$$

Hence for each $\phi \in L^{\infty}(0, T), g \in C_{c}^{1}\left(\mathbb{R}^{n} \mathbb{R}^{n}\right)$, we have

$$
\begin{aligned}
\int_{0}^{T} \int_{\mathbb{R}^{n}} \phi g^{i} \frac{u_{x_{i}}^{\epsilon}}{\left(\left|D u^{\epsilon}\right|^{2}+\epsilon^{2}\right)^{1 / 2}} H^{\epsilon}\left(\left|D u^{\epsilon}\right|^{2}+\epsilon^{2}\right)^{1 / 2} d x d t \\
\quad=\int_{0}^{T} \int_{\mathbb{R}^{n}} \phi\left(\delta_{i j}-\frac{u_{x_{i}}^{\epsilon} u_{x_{j}}^{\epsilon}}{\left(\left|D u^{\epsilon}\right|^{2}+\epsilon^{2}\right)^{1 / 2}}\right) g_{x_{j}}^{i}\left(\left|D u^{\epsilon}\right|^{2}+\epsilon^{2}\right)^{1 / 2} d x d t
\end{aligned}
$$

2. We wish to pass to limits as $\epsilon \rightarrow 0$. Now according to (4.3) and (4.4),

$$
\begin{gathered}
\int_{0}^{T} \int_{\mathbb{R}^{n} \cap\{|D u|>0\}} \phi\left(\delta_{i j}-\frac{u_{x_{i}}^{\epsilon} u_{x_{j}}^{\epsilon}}{\left(\left|D u^{\epsilon}\right|^{2}+\epsilon^{2}\right)}\right) g_{x_{j}}^{i}\left(\left|D u^{\epsilon}\right|^{2}+\epsilon^{2}\right)^{1 / 2} d x d t \\
\rightarrow \int_{0}^{T} \int_{\mathbb{R}^{n} \cap\{|D u|>0\}} \phi\left(\delta_{i j}-\frac{u_{x_{i}} u_{x_{j}}}{|D u|^{2}}\right) g_{x_{j}}^{i}|D u| d x d t .
\end{gathered}
$$


On the other hand,

$$
\begin{aligned}
& \left|\int_{0}^{T} \int_{\mathbb{R}^{n} \cap\{|D u|=0\}} \phi\left(\delta_{i j}-\frac{u_{x_{i}}^{\epsilon} u_{x_{j}}^{\epsilon}}{\left(\left|D u^{\epsilon}\right|^{2}+\epsilon^{2}\right)}\right) g_{x_{j}}^{i}\left(\left|D u^{\epsilon}\right|^{2}+\epsilon^{2}\right)^{1 / 2} d x d t\right| \\
& \quad \leq C \int_{0}^{T} \int_{\mathbb{R}^{n} \cap\{|D u|=0\} \cap s p t(g)}\left(\left|D u^{\epsilon}\right|^{2}+\epsilon^{2}\right)^{1 / 2} d x d t \\
& \quad \rightarrow 0, \text { according to (4.3). }
\end{aligned}
$$

Thus the right-hand side of (5.2) as $\epsilon \rightarrow 0$ converges to

$$
\int_{0}^{T} \int_{\mathbb{R}^{n}} \phi\left(\delta_{i j}-\frac{u_{x_{i}} u_{x_{j}}}{|D u|^{2}}\right) g_{x_{j}}^{i}|D u| d x d t
$$

the integrand interpreted as being zero on the set $\{|D u|=0\}$.

3. Employing (4.4) and (4.10) we see

$$
\begin{gathered}
\int_{0}^{T} \int_{\mathbb{R}^{n} \cap\{|D u|>0\}} \phi g^{i} \frac{u_{x_{i}}^{\epsilon}}{\left(\left|D u^{\epsilon}\right|^{2}+\epsilon^{2}\right)^{1 / 2}} H^{\epsilon}\left(\left|D u^{\epsilon}\right|^{2}+\epsilon^{2}\right)^{1 / 2} d x d t \\
\rightarrow \int_{0}^{T} \int_{\mathbb{R}^{n} \cap\{|D u|>0\}} \phi g^{i} \frac{u_{x_{i}}}{|D u|} H|D u| d x d t
\end{gathered}
$$

Additionally,

$$
\begin{aligned}
\left|\int_{0}^{T} \int_{\mathbb{R}^{n} \cap\{|D u|=0\}} \phi g^{i} \frac{u_{x_{i}}^{\epsilon}}{\left(\left|D u^{\epsilon}\right|^{2}+\epsilon^{2}\right)^{1 / 2}} H^{\epsilon}\left(\left|D u^{\epsilon}\right|^{2}+\epsilon^{2}\right)^{1 / 2} d x d t\right| & \left|H^{\epsilon}\right|\left(\left|D u^{\epsilon}\right|^{2}+\epsilon^{2}\right)^{1 / 2} d x d t \\
\leq & \left.C \int_{0}^{T} \int_{\mathbb{R}^{n} \cap|| D u|=0| \cap \operatorname{spt}(g)}\left(H^{\epsilon}\right)^{2}\left(\left|D u^{\epsilon}\right|^{2}+\epsilon^{2}\right)^{1 / 2} d x d t\right)^{1 / 2} \\
\leq & C\left(\int_{0}^{T} \int_{s p t(g)}\left(\left|D u^{\epsilon}\right|^{2}+\epsilon^{2}\right)^{1 / 2} d x d t\right)^{1 / 2} \\
& \times\left(\int_{0}^{T} \int_{\mathbb{R}^{n} \cap|| D u|=0| \cap s p t(g)}\left(\left|D u^{\epsilon}\right|^{2}+\epsilon^{2}\right)^{1 / 2} d x d t\right)^{1 / 2} \text { by }(4.5) \\
\leq & C\left(\int_{0}^{T} \int_{\mathbb{R}^{n} \cap\{|D u|=0 \mid n s p t(g)}\right. \\
\rightarrow & 0, \text { according to (4.3). }
\end{aligned}
$$

Consequently the left-hand side of (5.2) converges as $\epsilon \rightarrow 0$ to

$$
\int_{0}^{T} \int_{\mathbb{R}^{n}} \phi g^{i} \frac{u_{x_{i}}}{|D u|} H|D u| d x d t
$$

the integrand interpreted as being zero on the set $\{|D u|=0\}$. 
4. Thus

$$
\int_{0}^{T} \int_{\mathbb{R}^{n}} \phi g^{i} \frac{u_{x_{i}}}{|D u|} H|D u| d x d t=\int_{0}^{T} \int_{\mathbb{R}^{n}} \phi\left(\delta_{i j}-\frac{u_{x_{i}} u_{x_{j}}}{|D u|^{2}}\right) g_{x_{j}}^{i}|D u| d x d t
$$

for all $\phi \in L^{\infty}(0, T), g \in C_{c}^{1}\left(\mathbb{R}^{n} ; \mathbb{R}^{n}\right)$. Hence for a given vector field $g \in C_{c}^{1}\left(\mathbb{R}^{n} ; \mathbb{R}^{n}\right)$, equality (5.1) holds for a.e. $t \geq 0$. Choosing a countable dense subset of $C_{c}^{1}\left(\mathbb{R}^{n} ; \mathbb{R}^{n}\right)$ we conclude (5.1) holds for a.e. $t \geq 0$ and each $g \in C_{c}^{1}\left(\mathbb{R}^{n} ; \mathbb{R}^{n}\right)$.

Theorem 5.2. For all $0 \leq t_{1}<t_{2}<\infty$ and $\phi \in C_{c}^{1}\left(\mathbb{R}^{n}\right), \phi \geq 0$, we have the inequality

$$
\begin{gathered}
\left.\int_{\mathbb{R}^{n}} \phi|D u| d x\right|_{t=t_{2}}+\int_{t_{2}}^{t_{1}} \int_{\mathbb{R}^{n}} \phi|H|^{2}|D u|+D \phi \cdot \frac{D u}{|D u|} H|D u| d x d t \\
\leq\left.\int_{\mathbb{R}^{n}} \phi|D u| d x\right|_{t=t_{2}}
\end{gathered}
$$

where the integrands are interpreted as being zero on the set $\{|D u|=0\}$.

Proof. 1. Fix $\phi \in C_{c}^{\infty}\left(\mathbb{R}^{n}\right), \phi \geq 0$, and compute, as in the proof of Theorem 4.1,

$$
\begin{aligned}
\frac{d}{d t} \int_{\mathbb{R}^{n}} \phi\left(\left|D u^{\epsilon}\right|^{2}+\epsilon^{2}\right)^{1 / 2} d x= & \int_{\mathbb{R}^{n}} \phi \frac{D u^{\epsilon} \cdot D u_{t}^{\epsilon}}{\left(\left|D u^{\epsilon}\right|^{2}+\epsilon^{2}\right)^{1 / 2}} d x \\
= & -\int_{\mathbb{R}^{n}} \phi\left(H^{\epsilon}\right)^{2}\left(\left|D u^{\epsilon}\right|^{2}+\epsilon^{2}\right)^{1 / 2} d x \\
& -\int_{\mathbb{R}^{n}} \frac{D \phi \cdot D u^{\epsilon}}{\left(\left|D u^{\epsilon}\right|^{2}+\epsilon^{2}\right)^{1 / 2}} H^{\epsilon}\left(\left|D u^{\epsilon}\right|^{2}+\epsilon^{2}\right)^{1 / 2} d x
\end{aligned}
$$

Integrate in time from $t_{1}$ to $t_{2}$ :

$$
\begin{aligned}
\int_{\mathbb{R}^{n}} \phi\left(\left|D u^{\epsilon}\right|^{2}+\right. & \left.\epsilon^{2}\right)\left.^{1 / 2} d x\right|_{t=t_{2}}+\int_{t_{2}}^{t_{1}} \int_{\mathbb{R}^{n}} \phi\left(H^{\epsilon}\right)^{2}\left(\left|D u^{\epsilon}\right|^{2}+\epsilon^{2}\right)^{1 / 2} \\
+ & \frac{D \phi \cdot D u^{\epsilon}}{\left(\left|D u^{\epsilon}\right|^{2}+\epsilon^{2}\right)^{1 / 2}} H^{\epsilon}\left(\left|D u^{\epsilon}\right|^{2}+\epsilon^{2}\right)^{1 / 2} d x d t \\
& =\left.\int_{\mathbb{R}^{n}} \phi\left(\left|D u^{\epsilon}\right|^{2}+\epsilon^{2}\right)^{1 / 2} d x\right|_{t=t_{!}}
\end{aligned}
$$

2. Now (4.1) implies

$$
\left.\left.\int_{\mathbb{R}^{n}} \phi\left(\left|D u^{\epsilon}\right|^{2}+\epsilon^{2}\right)^{1 / 2} d x\right|_{t=t_{1}, t_{2}} \rightarrow \int_{\mathbb{R}^{n}} \phi|D u| d x\right|_{t=t_{1}, t_{2}}
$$


3. Furthermore,

$$
\begin{gathered}
\int_{t_{2}}^{t_{1}} \int_{\mathbb{R}^{n} \cap\{|D u|>0\}} D \phi \cdot \frac{D u^{\epsilon}}{\left(\left|D u^{\epsilon}\right|^{2}+\epsilon^{2}\right)^{1 / 2}} H^{\epsilon}\left(\left|D u^{\epsilon}\right|^{2}+\epsilon^{2}\right)^{1 / 2} d x d t \\
\rightarrow \int_{t_{2}}^{t_{1}} \int_{\left.\mathbb{R}^{\prime \prime} \cap|D u|>0\right\}} D \phi \cdot \frac{D u}{|D u|} H|D u| d x d t
\end{gathered}
$$

owing to (4.4) and (4.10). As in the previous proof,

$$
\int_{t_{2}}^{t_{1}} \int_{\mathbb{R}^{\prime \prime} \cap\{|D u|=0\}} D \phi \cdot \frac{D u^{\epsilon}}{\left(\left|D u^{\epsilon}\right|^{2}+\epsilon^{2}\right)^{1 / 2}} H^{\epsilon}\left(\left|D u^{\epsilon}\right|^{2}+\epsilon^{2}\right)^{1 / 2} d x d t \rightarrow 0
$$

Hence

$$
\begin{gathered}
\int_{t_{2}}^{t_{1}} \int_{\mathbb{R}^{n}} D \phi \\
\cdot \frac{D u^{\epsilon}}{\left(\left|D u^{\epsilon}\right|^{2}+\epsilon^{2}\right)^{1 / 2}} H^{\epsilon}\left(\left|D u^{\epsilon}\right|^{2}+\epsilon^{2}\right)^{1 / 2} d x d t \\
\rightarrow \int_{t_{2}}^{t_{1}} \int_{\mathbb{R}^{n}} D \phi \cdot \frac{D u}{|D u|} H|D u| d x d t
\end{gathered}
$$

the integrand being zero on $\{|D u|=0\}$.

4. We next claim

$$
\int_{t_{2}}^{t_{1}} \int_{\mathbb{R}^{n}} \phi H^{2}|D u| d x d t \leq \liminf _{\epsilon \rightarrow 0} \int_{t_{2}}^{t_{1}} \int_{\mathbb{R}^{n}} \phi\left(H^{\epsilon}\right)^{2}\left(\left|D u^{\epsilon}\right|^{2}+\epsilon^{2}\right)^{1 / 2} d x d t .
$$

To prove this let us observe for each $\psi \in L^{\infty}\left(\mathbb{R}^{n} \times(0, \infty)\right)$,

$$
\begin{aligned}
\int_{t_{2}}^{t_{1}} \int_{\mathbb{R}^{n}} \phi \psi H|D u| d x d t= & \lim _{\epsilon \rightarrow 0} \int_{t_{2}}^{t_{1}} \int_{\mathbb{R}^{n}} \phi \psi H^{\epsilon}\left(\left|D u^{\epsilon}\right|^{2}+\epsilon^{2}\right)^{1 / 2} d x d t \\
\leq & \liminf _{\epsilon \rightarrow 0}\left(\int_{t_{2}}^{t_{1}} \int_{\mathbb{R}^{n}} \phi\left(H^{\epsilon}\right)^{2}\left(\left|D u^{\epsilon}\right|^{2}+\epsilon^{2}\right)^{1 / 2} d x d t\right)^{1 / 2} \\
& \left(\int_{t_{2}}^{t_{1}} \int_{\mathbb{R}^{n}} \phi \psi^{2}|D u| d x d t\right)^{1 / 2} .
\end{aligned}
$$

Set $\psi=\rho_{m}(H)$, where

$$
\rho_{m}(z)= \begin{cases}m & z \geq m \\ z & -m \leq z \leq m \\ -m & z \leq-m\end{cases}
$$


to deduce

$$
\left(\int_{t_{2}}^{t_{1}} \int_{\mathbb{R}^{n}} \phi H \rho_{m}(H)|D u| d x d t\right)^{1 / 2} \leq \liminf _{\epsilon \rightarrow 0}\left(\int_{t_{2}}^{t_{1}} \int_{\mathbb{R}^{n}} \phi\left(H^{\epsilon}\right)^{2}\left(\left|D u^{\epsilon}\right|^{2}+\epsilon^{2}\right)^{1 / 2} d x d t\right)^{1 / 2},
$$

since $\left|\rho_{m}(H)\right| \leq|H|$. Let $m \rightarrow \infty$ and apply the Monotone Convergence Theorem.

5. Inequality (5.3) now follows from (5.5), (5.6), and (5.7).

\section{Interpreting $H$ as mean curvature}

Our intention in this section and the next is to interpret (a) formula (5.1) as saying $H$ (defined by (4.9)) is the mean curvature of the level sets of $u$, and (b) formula (5.3) as saying these level sets are evolving by mean curvature motion. First we demonstrate that a.e. level set of $u$ for a.e. time intersects $\{|D u|=0\}$ only on a negligible set.

Lemma 6.1. For a.e. $(\gamma, t) \in \mathbb{R} \times(0, \infty)$,

$H^{n-1}\left(\left\{x \in \mathbb{R}^{n} \mid u(x, t)=\gamma, u\right.\right.$ is not differentiable at $(x, t)$ or $\left.\left.|D u(x, t)|=0\right\}\right)=0$.

(Recall our notation: $D u=D_{x} u=\left(u_{x_{1}}, \cdots, u_{x_{n}}\right)$ is the gradient taken with respect to $x \in \mathbb{R}^{n}$.)

Proof. Define the Lipschitz function $v: \mathbb{R}^{n} \times(0, \infty) \rightarrow \mathbb{R}^{2}$ by

$$
v(x, t) \equiv(u(x, t), t) \quad\left(x \in \mathbb{R}^{n}, t>0\right) .
$$

Then the full gradient of $v$ is

$$
D_{x, t} v=\left(\begin{array}{cc}
u_{x_{1}} \ldots u_{x_{n}} & u_{t} \\
0 \ldots 0 & 1
\end{array}\right)_{2 \times(n+1)} \text { a.e. in } \mathbb{R}^{n} \times(0, \infty),
$$

and so the Jacobian of $v$ is

$$
\begin{aligned}
J v & =\left(\operatorname{det}\left(D_{x, t} v \cdot\left(D_{x, t} v\right)^{T}\right)\right)^{1 / 2} \\
& =\left(\operatorname{det}\left(\begin{array}{cc}
|D u|^{2}+u_{t}^{2} & u_{t} \\
u_{t} & 1
\end{array}\right)\right)^{1 / 2} \\
& =|D u| \text { a.e. in } \mathbb{R}^{n} \times(0, \infty)
\end{aligned}
$$

The Coarea Formula (Federer $[11, \$ 3.2 .11])$ asserts that

$$
\int_{A} J v d x d t=\int_{\mathbb{R}^{2}} H^{(n+1)-2}\left(A \cap v^{-1}\{z\}\right) d z
$$


for each Lebesgue measurable set $A \subseteq \mathbb{R}^{n} \times(0, \infty)$. Substituting $J v=|D u|$ and $A=$ $\{u$ is not differentiable or $|D u|=0\}$, we deduce

$$
H^{n-1}\left(A \cap v^{-1}\{z\}\right)=0 \text { for a.e. } z=(\gamma, t) \in \mathbb{R}^{2} .
$$

Notation. Let us henceforth set

$$
v \equiv \frac{D u}{|D u|} \text { on }\{|D u|>0\}
$$

Also, for each $\gamma \in \mathbb{R}, t \geq 0$, we write

$$
\Gamma_{t}^{\gamma} \equiv\left\{x \in \mathbb{R}^{n} \mid u(x, t)=\gamma\right\}
$$

to denote the level set $\{u=\gamma\}$ at time $t$. We know

$$
H^{n-1}\left(\Gamma_{t}^{\gamma}\right)<\infty
$$

and also $\Gamma_{r}^{\gamma}$ is $\left(H^{n-1}, n-1\right)$-rectifiable for a.e. $(\gamma, t)$, according to [10, Lemma 2.2]. Furthermore, Lemma 6.1 says

$$
\left.\begin{array}{l}
\text { for a.e. }(\gamma, t) \\
H^{n-1}\left(\Gamma_{t}^{\gamma} \cap\{|D u(\cdot, t)|=0\}\right)=0 .
\end{array}\right\}
$$

We next decompose formula (5.1) into a corresponding equality for a.e. level set:

Theorem 6.2. For a.e. $(\gamma, t)$ we have the equality

$$
\int_{\Gamma_{t}^{\gamma}}\left(\delta_{i j}-v_{i} v_{j}\right) g_{x_{j}}^{i} d H^{n-1}=\int_{\Gamma_{t}^{\gamma}} g^{i} v_{i} H d H^{n-1}
$$

for each vector field $g \in C_{c}^{1}\left(\mathbb{R}^{n} ; \mathbb{R}^{n}\right), g=\left(g^{1}, \ldots, g^{n}\right)$.

Proof. 1. Owing to (5.1),

$$
\int_{0}^{T} \int_{\mathbb{R}^{n}} \phi\left(\delta_{i j}-v_{i} v_{j}\right) g_{x_{j}}^{i}|D u| d x d t=\int_{0}^{T} \int_{\mathbb{R}^{n}} \phi g^{i} v_{i} H|D u| d x d t
$$

for each $T>0, \phi \in L^{\infty}(0, T), g \in C_{c}^{1}\left(\mathbb{R}^{n} ; \mathbb{R}^{n}\right)$. By approximation, the equality obtains if $g$ is Lipschitz, with compact support. 
Let $\Phi: \mathbb{R} \rightarrow \mathbb{R}$ be any smooth function. Then replacing $g$ by $\Phi(u) g$ in (6.6) we find

$$
\int_{0}^{T} \int_{\mathbb{R}^{n}} \phi\left(\delta_{i j}-v_{i} v_{j}\right)\left(\Phi(u) g^{i}\right)_{x_{j}}|D u| d x d t=\int_{0}^{T} \int_{\mathbb{R}^{n}} \phi \Phi(u) g^{i} v_{i} H|D u| d x d t
$$

Now on the set $\{|D u|>0\}$,

$$
\begin{aligned}
\left(\delta_{i j}-v_{i} v_{j}\right)\left(\Phi(u) g^{i}\right)_{x_{j}} & =\Phi(u)\left(\delta_{i j}-v_{i} v_{j}\right) g_{x_{j}}^{i}+\Phi^{\prime}(u)\left(\delta_{i j}-v_{i} v_{j}\right) u_{x_{j}} g^{i} \\
& =\Phi(u)\left(\delta_{i j}-v_{i} v_{j}\right) g_{x_{j}}^{i}
\end{aligned}
$$

since $\left(\delta_{i j}-v_{i} v_{j}\right) u_{x_{j}} g^{i}=0$. Hence $(6.7)$ becomes

$$
\int_{0}^{T} \int_{\mathbb{R}^{n}} \phi \Phi(u)\left(\delta_{i j}-v_{i} v_{j}\right) g^{i} x_{j}|D u| d x d t=\int_{0}^{T} \int_{\mathbb{R}^{n}} \phi \Phi(u) g^{i} v_{i} H|D u| d x d t
$$

and so, according to the Coarea Formula,

$$
\begin{aligned}
\int_{0}^{T} \int_{\mathbb{R}} \phi(t) & \Phi(\gamma)\left(\int_{\Gamma_{t}^{\gamma}}\left(\delta_{i j}-v_{i} v_{j}\right) g_{x_{j}}^{i} d H^{n-1}\right) d \gamma d t \\
= & \int_{0}^{T} \int_{\mathbb{R}} \phi(t) \Phi(\gamma)\left(\int_{\Gamma_{t}^{\gamma}} g^{i} v_{i} H d H^{n-1}\right) d \gamma d t
\end{aligned}
$$

This equality obtains for each $\phi \in L^{\infty}(0, T)$ and $\Phi \in C^{\infty}(\mathbb{R})$; whence (6.5) follows for a.e. $(\gamma, t)$.

Remark. As in Allard [1, §4.1(2)], we have

$$
\left.\frac{d}{d s} H^{n-1}\left(h\left(s, \Gamma_{t}^{\gamma}\right)\right)\right|_{s=0}=\int_{\Gamma_{t}^{\gamma}}\left(\delta_{i j}-v_{i} v_{j}\right) g_{x_{j}}^{i} d H^{n-1}
$$

where $h: \mathbb{R}^{n} \times(-1,1) \rightarrow \mathbb{R}^{n}$ is smooth,

$$
h(0, x)=x, \frac{\partial h}{\partial s}(0, x)=g(x) \quad\left(x \in \mathbb{R}^{n}\right)
$$

The left-hand side of (6.8) is thus the first variation of the $(n-1)$-dimensional area of $\Gamma_{t}^{\gamma}$ under the vector field $g$. Theorem 6.2 asserts the first variation equals $\int_{\Gamma_{t}^{\gamma}} g \cdot \nu H d H^{n-1}$ for a.e. $(\gamma, t)$; so that $-H v$ can be interpreted geometrically as the mean curvature vector. Note further from Lemma 4.3, $\int_{\Gamma_{t}^{\gamma}} H^{2} d H^{n-1}<\infty$ for a.e. $(\gamma, t)$. 
Finally we decompose inequality (5.3) into constituent inequalities for a.e. level set.

Theorem 6.3. For a.e. $\gamma \in \mathbb{R}$ we have for a.e. $0 \leq t_{1}<t_{2}<\infty$ the inequality

$$
\int_{\Gamma_{t_{2}}^{\gamma}} \phi d H^{n-1}+\int_{t_{2}}^{t_{1}} \int_{\Gamma_{t}^{\gamma}} \phi H^{2}+D \phi \cdot \nu H d H^{n-1} d t \leq \int_{\Gamma_{t_{1}^{\gamma}}^{\gamma}} \phi d H^{n-1}
$$

for all $\phi \in C_{c}^{1}\left(\mathbb{R}^{n}\right), \phi \geq 0$.

Proof. 1. Let $\Phi: \mathbb{R} \rightarrow \mathbb{R}$ be smooth, with $\Phi^{\prime}>0$. Owing to [8, Theorem 2.8], $\tilde{u} \equiv \Phi(u)$ is the unique weak solution of the mean curvature evolution PDE

$$
\left.\begin{array}{ll}
\tilde{u}_{t}=\left(\delta_{i j}-\frac{\tilde{u}_{x_{i}} \tilde{x}_{x_{j}}}{|D \tilde{u}|^{2}}\right) \tilde{u}_{x_{i} x_{j}} & \text { in } \mathbb{R}^{n} \times(0, \infty) \\
\tilde{u}=\tilde{g} & \text { in } \mathbb{R}^{n} \times\{t=0\},
\end{array}\right\}
$$

for $\tilde{g} \equiv \Phi(g)$. Since $\Phi^{\prime}>0$, we check using Lemma 2.1

$$
\sup _{0<\epsilon \leq 1} \int_{\mathbb{R}^{n}}\left|\operatorname{div}\left(\frac{D \tilde{g}}{\left(|D \tilde{g}|^{2}+\epsilon^{2}\right)^{1 / 2}}\right)\right| d x<\infty .
$$

Applying the theory developed before to $\tilde{u}$ in place of $u$ we find that

$$
\int_{\Gamma_{t}^{\gamma}}\left(\delta_{i j}-\tilde{v}_{i} \tilde{v}_{j}\right) g_{x_{j}}^{i} d H^{n-1}=\int_{\Gamma_{t}^{\gamma}} g^{i} \tilde{v}_{i} \tilde{H} d H^{n-1}
$$

for a.e. $(\gamma, t)$, all $g \in C_{c}^{1}\left(\mathbb{R}^{n} ; \mathbb{R}^{n}\right)$, and

$$
\left.\int_{\mathbb{R}^{n}} \phi|D \tilde{u}| d x\right|_{t=t_{2}}+\int_{t_{2}}^{t_{1}} \int_{\mathbb{R}^{n}} \phi(\tilde{H})^{2}|D \tilde{u}|+D \phi \cdot \tilde{v} \tilde{H}|D \tilde{u}| d x d t \leq\left.\int_{\mathbb{R}^{n}} \phi|D \tilde{u}| d x\right|_{t=t_{1}}
$$

for all $0 \leq t_{1}<t_{2}<\infty$ and all $\phi \in C_{c}^{1}\left(\mathbb{R}^{n}\right), \phi \geq 0$. But since $\tilde{u}=\Phi(u)$ and $\Phi^{\prime}>0$,

$$
\tilde{v}=v=D u|D u|, \tilde{H}=H=\frac{u_{t}}{|D u|} \text { a.e. on }\{|D u|>0\}=\{|D \tilde{u}|>0\}
$$

Thus

$$
\begin{gathered}
\left.\int_{\mathbb{R}^{n}} \Phi^{\prime}(u) \phi|D u| d x\right|_{t=t_{2}}+\int_{t_{2}}^{t_{1}} \int_{\mathbb{R}^{n}} \Phi^{\prime}(u)\left(\phi H^{2}+D \phi \cdot v H\right)|D u| d x d t \\
\leq\left.\int_{\mathbb{R}^{n}} \Phi^{\prime}(u) \phi|D u| d x\right|_{t=t_{2}} .
\end{gathered}
$$


One final time we employ the Coarea formula and deduce

$$
\begin{gathered}
\int_{\mathbb{R}} \Phi^{\prime}(\gamma)\left(\int_{\Gamma_{t_{2}}^{\gamma}} \phi d H^{n-1}\right) d \gamma+\int_{\mathbb{R}} \Phi^{\prime}(\gamma)\left(\int_{t_{1}}^{t_{2}} \int_{\Gamma_{t}^{\gamma}} \phi H^{2}+D \phi \cdot v d H^{n-1} d t\right) d \gamma \\
\leq \int_{\mathbb{R}} \Phi^{\prime}(\gamma)\left(\int_{\Gamma_{t}^{\gamma}} \phi d H^{n-1}\right) d \gamma
\end{gathered}
$$

This inequality is valid for all $0 \leq t_{1}<t_{2}<\infty$, all $\phi \in C_{c}^{1}\left(\mathbb{R}^{n}\right), \phi \geq 0$, and each smooth positive function $\Phi^{\prime}$ : the theorem follows.

\section{Varifolds and varifolds moving by mean curvature}

Let us now quickly recall the standard terminology for the theory of varifolds, from Allard [1], Brakke [2], and Simon [21].

Varifolds. Let $G(n, n-1)$ denote the Grassman manifold of unoriented, (n-1)-dimensional planes in $\mathbb{R}^{n}$. If $S \in G(n, n-1)$, we will use the symbol $S$ to denote also the orthogonal projection of $\mathbb{R}^{n}$ onto $S$. Write $A \cdot B=\operatorname{trace}\left(A^{t} \cdot B\right)$ for linear mappings $A, B: \mathbb{R}^{n} \rightarrow \mathbb{R}^{n}$.

We say $V$ is an $(n-1)$-dimensional varifold in $\mathbb{R}^{n}$ if $V$ is a Radon measure on $G_{n-1}\left(\mathbb{R}^{n}\right) \equiv$ $\mathbb{R}^{n} \times G(n, n-1) . V_{n-1}\left(\mathbb{R}^{n}\right)$ denotes the collection of all $(n-1)$-dimensional varifolds in $\mathbb{R}^{n}$. If $V \in V_{n-1}\left(\mathbb{R}^{n}\right)$, we define its weight $\|V\|$ to be the Randon measure on $\mathbb{R}^{n}$ given by

$$
\|V\|(A)=V\{(x, S) \mid x \in A, S \in G(n, n-1)\}
$$

for each Borel set $A \subset \mathbb{R}^{n}$.

If $\Gamma$ is an $\left(H^{n-1}, n-1\right)$-rectifiable subset of $\mathbb{R}^{n}$ (cf. Federer $\left.[11, \S 3.2 .14]\right)$, we associate the corresponding varifold $v(\Gamma) \in V_{n-1}\left(\mathbb{R}^{n}\right)$ defined by

$$
v(\Gamma)(A)=H^{n-1}\left\{x \in \mathbb{R}^{n} \mid\left(x, \operatorname{Tan}^{n-1}\left(H^{n-1}\llcorner\Gamma, x)\right) \in A\right\}\right.
$$

for each Borel $A \subset G_{n-1}\left(\mathbb{R}^{n}\right)$. Here $\operatorname{Tan}^{n-1}\left(H^{n-1}\llcorner\Gamma, x)\right.$ is for a.e. $x \in \Gamma$ the approximate tangent space to $\Gamma$ at $x$. We say $V \in V_{n-1}\left(\mathbb{R}^{n}\right)$ is an $(n-1)$-dimensional rectifiable varifold if there exist positive real numbers $\left\{c_{k}\right\}_{k=1}^{\infty}$ and $\left(H^{n-1}, n-1\right)$-rectifiable sets $\left\{\Gamma_{k}\right\}_{k=1}^{\infty}$ such that

$$
V=\sum_{k=1}^{\infty} c_{k} v\left(\Gamma_{k}\right)
$$

If the $\left\{c_{k}\right\}_{k=1}^{\infty}$ can be selected to be nonnegative integers, we say $V$ is an $(n-1)$-dimensional integral varifold. We denote by $R V_{n-1}\left(\mathbb{R}^{n}\right)$ (resp. $I V_{n-1}\left(\mathbb{R}^{n}\right)$ ) the collection of $(n-1)$-dimensional rectifiable (resp. integral) varifolds in $\mathbb{R}^{n}$. 
First variation. If $V \in V_{n-1}\left(\mathbb{R}^{n}\right)$, we define its first variation

$$
\delta V(g)=\int D g(x) \cdot S d V(x, S)
$$

for each vector field $g \in C_{c}^{1}\left(\mathbb{R}^{n} ; \mathbb{R}^{n}\right)$. The corresponding total variation is the measure $\|\delta V\|$ defined by

$$
\|\delta V\|(G)=\sup \left\{\delta V(g)\left|g \in C_{c}^{1}\left(\mathbb{R}^{n} ; \mathbb{R}^{n}\right), \operatorname{spt}(g) \subset C,\right| g \mid \leq 1\right\}
$$

for open sets $G \subset \mathbb{R}^{n}$. If $\|\delta V\|$ is a Radon measure and is absolutely continuous with respect to $\|V\|$ (in which case we write $\|\delta V\|_{\text {sing }}=0$ ), there exists a $\|V\|$-measurable function $h(V, x): \mathbb{R}^{n} \rightarrow \mathbb{R}^{n}$ such that

$$
\delta V(g)=-\int g(x) \cdot h(V, x) d\|V\|(x)
$$

for all $g$ as above; $h(V, \cdot)$ is the generalized mean curvature vector. More generally, if $\phi \in C_{c}^{1}\left(\mathbb{R}^{n}\right)$, let us write

$$
\delta(V, \phi)(g)=-\int h(V, x) \cdot g(x) \phi(x) d\|V\|(x)+\int S^{\perp}(D \phi(x)) \cdot g(x) d V(x, S),
$$

$S^{\perp}$ denoting the projection onto the line perpendicular to $S$. In particular, we set

$$
\begin{aligned}
\delta(V, \phi)(h(V, \cdot))= & -\int|h(V, x)|^{2} \phi(x) d\|V\|(x) \\
& +\int S^{\perp}(D \phi(x)) \cdot h(V, x) d V(x, S)
\end{aligned}
$$

if $\|\delta V\|$ is a Radon measure with $\|\delta V\|_{\text {sing }}=0, \int|h(V, x)|^{2} \phi(x) d\|V\|(x)<\infty$. Otherwise, set

$$
\delta(V, \phi)(h(V, \cdot))=-\infty
$$

Varifolds moving by mean curvature. Consider a family $\left\{V_{t}\right\}_{t \geq 0}$, where $V_{t} \in V_{n-1}\left(\mathbb{R}^{n}\right)$ for each $t \geq 0$. Following Brakke $[2, \S 3.3]$ we define $\left\{V_{t}\right\}_{t \geq 0}$ to be an $(n-1)$-dimensional varifold moving by its mean curvature provided

$$
\bar{D}\left\|V_{t}\right\|(\phi) \leq \delta\left(V_{t}, \phi\right)\left(h\left(V_{t}, \cdot\right)\right)
$$

for each $\phi \in C_{c}^{1}\left(\mathbb{R}^{n}\right), \phi \geq 0$, and each time $t \geq 0$. Here $\bar{D}\left\|V_{t}\right\|(\phi)$ denotes the upper derivate $\lim _{\sup _{s \rightarrow t}} \frac{\left\|V_{t}\right\|(\phi)-\left\|V_{s}\right\|(\phi)}{t-s}$. We say $\left\{V_{t}\right\}_{t \geq 0}$ is a unit density varifold moving by its mean curvature if in addition for a.e. $t>0$,

$$
\Theta^{n-1}\left(\left\|V_{t}\right\|, x\right)=1 \text { for }\left\|V_{t}\right\| \text { a.e. } x \in \mathbb{R}^{n},
$$


where

$$
\Theta^{n-1}\left(\left\|V_{t}\right\|, x\right)=\lim _{r \rightarrow 0^{+}} \frac{\left\|V_{t}\right\|(B(x, r))}{\alpha(n-1) r^{n-1}}
$$

is the (n-1)-dimensional density of $\left\|V_{t}\right\|$ at $x \in \mathbb{R}^{n}$.

See Brakke [2] for much more explanation about these definitions.

Level sets. Our intention next is to interpret a.e. level set of our solution $u$ of the mean curvature evolution PDE (2.4) as comprising a unit density varifold moving by its mean curvature.

For this, let us recall Section 6 and hereafter fix an index $\gamma \in \mathbb{R}$ for which the corresponding level sets

$$
\Gamma_{t}^{\gamma}=\left\{x \in \mathbb{R}^{n} \mid u(x, t)=\gamma\right\} \quad(t \geq 0)
$$

satisfy

$$
H^{n-1}\left(\Gamma_{t}^{\gamma}\right) \leq H^{n-1}\left(\Gamma_{0}^{\gamma}\right)
$$

$\left.\begin{array}{l}\Gamma_{t} \text { is }\left(H^{n-1}, n-1\right) \text { rectifiable, with } \\ \text { approximate normal } \nu=\frac{D u}{|D u|} \quad H^{n-1} \text { a.e., }\end{array}\right\}$

$$
\int_{\Gamma_{t}^{\gamma}} H^{2} d H^{n-1}<\infty
$$

$$
\begin{aligned}
& \int_{\Gamma_{t}^{\gamma}}\left(\delta_{i j}-v_{i} v_{j}\right) g_{x_{j}}^{i} d H^{n-1}=\int_{\Gamma_{r}^{\gamma}} g^{i} v_{i} H d H^{n-1} \\
& \text { for all } g \in C_{c}^{1}\left(\mathbb{R}^{n} ; \mathbb{R}^{n}\right),
\end{aligned}
$$

and

$$
\left.\begin{array}{l}
\int_{\Gamma_{r_{2}}^{\gamma}} \phi d H^{n-1}+\int_{t_{1}}^{t_{2}} \int_{\Gamma_{t}^{\gamma}} \phi H^{2}+D \phi \cdot v H d H^{n-1} d t \leq \int_{\Gamma_{t_{1}}^{\gamma}} \phi d H^{n-1} \\
\text { for all } g \in C_{c}^{1}\left(\mathbb{R}^{n} ; \mathbb{R}^{n}\right), \phi \geq 0
\end{array}\right\}
$$

for a.e. $t, t_{1}, t_{2} \geq 0, t_{1}<t_{2}$.

Let $\mathcal{T}$ denote the set of times $t \in[0, \infty)$ such that (7.5)-(7.9) are valid for all $t, t_{1}, t_{2} \in \mathcal{T}$, $t_{1}<t_{2}$. Then

$$
|[0, \infty)-\mathcal{T}|=0
$$


Definition of $\left\{V_{t}^{\gamma}\right\}_{t \geq 0}$. Our aim is to associate with the level sets $\left\{\Gamma_{t}^{\gamma}\right\}_{t \geq 0}$ a unit-density varifold $\left\{V_{t}^{\gamma}\right\}_{t \geq 0}$ moving by its mean curvature. We begin by setting

$$
V_{t}^{\gamma}=v\left(\Gamma_{t}^{\gamma}\right) \text { for } t \in \mathcal{T}
$$

Thus $V_{t}^{\gamma}$ is for $t \in \mathcal{T}$ a unit density, rectifiable varifold, with

$$
\begin{gathered}
\left\|V_{t}^{\gamma}\right\|=H^{n-1}\left\llcorner\Gamma_{t}^{\gamma},\right. \\
h\left(V_{t}^{\gamma}, \cdot\right)=-H \nu \quad H^{n-1} \text { a.e. on } \Gamma_{t}^{\gamma},
\end{gathered}
$$

and

$$
\delta\left(V_{t}, \phi\right)\left(h\left(V_{t}^{\gamma}, \cdot\right)\right)=-\int_{\Gamma_{t}^{\gamma}} H^{2} \phi+D \phi \cdot v H d H^{n-1}
$$

for $\phi \in C_{c}^{1}\left(\mathbb{R}^{n}\right)$.

We must now extend the definition (7.10) to times $t \notin \mathcal{T}$. Following Brakke $[2, \S 4.14]$ we first note using (7.5), (7.9), and (7.11) that if $t_{1}, t_{2} \in \mathcal{T}, t_{1}<t_{2}$,

$$
\begin{aligned}
\left\|V_{t_{2}}^{\gamma}\right\|(\phi)-\left\|V_{t_{1}}^{\gamma}\right\|(\phi) & \leq-\int_{t_{1}}^{t_{2}} \int_{\Gamma_{t}^{\gamma}} H^{2} \phi+D \phi \cdot v H d H^{n-1} d t \\
& \leq \int_{t_{1}}^{t_{2}} \int_{\Gamma_{t}^{\gamma}}|D \phi|^{2} \phi d H^{n-1} d t \\
& \leq K\left(t_{2}-t_{1}\right)
\end{aligned}
$$

for

$$
K=\left(\sup _{\mathbb{R}^{n}} \frac{|D \phi|^{2}}{\phi}\right) H^{n-1}\left(\Gamma_{0}^{\gamma}\right)<\infty
$$

Thus the mapping $t \mapsto\left\|V_{t}^{\gamma}\right\|(\phi)-K t$ is nonincreasing on the set $\mathcal{T}$; therefore, if $t>0, t \notin \mathcal{T}$, the limit

$$
\left\|V_{t}^{\gamma}\right\|(\phi)=\lim _{\substack{s \rightarrow t^{-} \\ s \in \mathcal{T}}}\left\|V_{s}^{\gamma}\right\|(\phi)
$$

exists for all $\phi \in C_{c}^{1}\left(\mathbb{R}^{n}\right), \phi \geq 0$. In particular, we can define the Radon measure $\left\|V_{t}^{\gamma}\right\|$ for $t \notin \mathcal{T}$ by declaring

$$
\left\|V_{t}^{\gamma}\right\|=\lim _{\substack{s \rightarrow t^{-} \\ s \in \mathcal{T}}}\left\|V_{s}^{\gamma}\right\|
$$


the limit taken in the sense of weak convergence of Radon measures. Recalling (7.10) and (7.13), we deduce

$$
\left\|V_{t_{2}}^{\gamma}\right\|(\phi) \leq\left\|V_{t_{1}}^{\gamma}\right\|(\phi)+\int_{t_{1}}^{t_{2}} \delta\left(V_{t}^{\gamma}, \phi\right)\left(h\left(V_{t}^{\gamma}, \cdot\right)\right) d t
$$

for all times $0 \leq t_{1} \leq t_{2}<\infty$ and nonnegative test functions $\phi$ as above. Now (7.17) defines the measure $\left\|V_{t}^{\gamma}\right\|$ for all times $t \notin \mathcal{T}$, and we must extend this to define the varifold $V_{t}^{\gamma}$ for $t \notin \mathcal{T}$. As in Brakke [2, \$4.26], if $t \notin \mathcal{T}$ we define $V_{t}^{\gamma} \in V_{n-1}\left(\mathbb{R}^{n}\right)$ by setting

$$
\begin{aligned}
V_{t}^{\gamma}(A)= & \left\|V_{t}\right\|\left(\left\{x \in \mathbb{R}^{n} \mid\left(x, \operatorname{Tan}^{n-1}\left(\left\|V_{t}\right\|, x\right)\right) \in A\right\}\right) \\
+ & \left\|V_{t}\right\|\left(\left\{x \in \mathbb{R}^{n} \mid \operatorname{Tan}^{k-1}\left(\left\|V_{t}\right\|, x\right) \notin G(n, n-1)\right.\right. \text { and } \\
& (x, T) \in A \text { for some } T \in G(n, n-1)\})
\end{aligned}
$$

for each Borel set $A \subset G_{n-1}\left(\mathbb{R}^{n}\right)$. Here

$$
\operatorname{Tan}^{n-1}\left(\left\|V_{t}\right\|, x\right)
$$

is the approximate $(n-1)$-dimensional tangent space to spt $\left\|V_{t}\right\|$ at $x$. Owing to Allard [1, $\$ 3.5(1)$ (a)] the second term on the right-hand side of (7.19) is zero, provided $V_{t}^{\gamma}$ is rectifiable.

Theorem 7.1. Define $V_{t}^{\gamma}$ by (7.10) if $t \in \mathcal{T}$, and by (7.17), (7.19) if $t \notin \mathcal{T}$. Then $\left\{V_{t}^{\gamma}\right\}_{t \geq 0}$ is a unit-density varifold moving by its mean curvature.

Our proof closely follows Brakke $[2, \S 4.28,4.29]$.

Proof. 1. Fix $\phi \in C_{c}^{1}\left(\mathbb{R}^{n}\right), \phi \geq 0$. According to (7.3) we must verify

$$
\bar{D}\left\|V_{t}^{\gamma}\right\|(\phi) \leq \delta\left(V_{t}^{\gamma}, \phi\right)\left(h\left(V_{t}^{\gamma}, \cdot\right)\right)
$$

for each time $t \geq 0$. If $\bar{D}\left\|V_{t}^{\gamma}\right\|(\phi)=-\infty$, we are done, and so we may as well suppose

$$
\bar{D}\left\|V_{t}^{\gamma}\right\|(\phi)>-\infty
$$

2. Assume in fact

$$
D^{-}\left\|V_{t}^{\gamma}\right\|(\phi)>-\infty
$$

$D^{-}$denoting the upper left derivate. 
For each $0<B<\infty$, we define

$$
E_{B}=\left\{r \in \mathcal{T} \mid \delta\left(V_{r}^{\gamma}, \phi\right)\left(h\left(V_{r}^{\gamma}, \cdot\right)\right)>-B\right\} .
$$

Note also, as in (7.14),

$$
\delta\left(V_{r}^{\gamma}, \phi\right)\left(h\left(V_{r}^{\gamma}, \cdot\right)\right) \leq K \text { for a.e. } r \geq 0,
$$

$K$ defined by (7.15). Thus if $0<s<t,(7.18)$ yields

$$
\begin{aligned}
\left\|V_{t}^{\gamma}\right\|(\phi)-\left\|V_{s}^{\gamma}\right\|(\phi) & \leq \int_{s}^{t} \delta\left(V_{r}^{\gamma}, \phi\right)\left(h\left(V_{r}^{\gamma}, \cdot\right)\right) d r \\
& \leq \int_{[s, t]-E_{B}}-B d r+\int_{E_{B}} K d r
\end{aligned}
$$

Rearranging we deduce

$$
\limsup _{s \rightarrow t^{-}} \frac{\left|[s, t]-E_{B}\right|}{t-s} \leq-\left[K-D^{-}\left\|V_{t}^{\gamma}\right\|(\phi)\right] .
$$

3. Suppose $t_{k} \in[0, t) \cap E_{B}(k=1,2, \ldots), t_{k} \rightarrow t^{-}$. Then (7.13), (7.23) easily imply the bound

$$
\sup _{k}\left\|\delta V_{t_{k}}^{\gamma}\right\|\left(W_{\eta}\right)<\infty
$$

where $\eta>0, W_{\eta}=\left\{x \in \mathbb{R}^{n} \mid \phi(x) \geq \eta>0\right\}$. Since

$$
\sup _{k}\left\|V_{t_{k}}^{\gamma}\right\|\left(W_{\eta}\right)<\infty
$$

Allard's Compactness Theorem for Integral Varifolds $[1, \S 6.4]$ implies $\left\{V_{t_{k}}^{\gamma}\left\llcorner W_{\eta}\right\}_{k=1}^{\infty}\right.$ is precompact in $I V_{n-1}\left(\mathbb{R}^{n}\right)$. Thus, passing to a subsequence if necessary and reindexing, we have

$$
V_{t_{k}}^{\gamma}\left\llcornerW _ { \eta } \rightarrow U _ { t } \left\llcorner W_{\eta}\right.\right.
$$

for some $U_{t} \in I V_{n-1}\left(\mathbb{R}^{n}\right)$. In particular,

$$
\left\|V_{t_{k}}^{\gamma}\right\|_{\llcorner} W_{\eta} \rightarrow\left\|U_{t}\right\|\left\llcorner W_{\eta}\right.
$$

But

$$
\left\|V_{t_{k}}^{\gamma}\right\| \rightarrow\left\|V_{t}^{\gamma}\right\|
$$

according to (7.17) $W_{\eta}=\left\|V_{t}^{\gamma}\right\|\left\llcorner W_{\eta}\right.$. Thus $V_{t}^{\gamma}\left\llcorner W_{\eta}\right.$ is an integral varifold. This conclusion obtains 
for each $\eta>0$ and so

$$
V_{t}\left\llcorner\{\phi 0\} \in I V_{n-1}\left(\mathbb{R}^{n}\right)\right.
$$

4. Now Brakke $[2, \S 4.28]$ utilizes (7.27) to establish the upper semicontinuity assertion:

$$
\limsup _{k \rightarrow \infty} \delta\left(V_{t_{k}}^{\gamma}, \phi\right)\left(h\left(V_{t_{k}}^{\gamma}, \cdot\right)\right) \leq \delta\left(V_{t}, \phi\right)\left(h\left(V_{t}, \cdot\right)\right)
$$

Fix $\eta>0$, and then choose $B$ so large

$$
\frac{K-D^{-}\left\|V_{t}^{\gamma}\right\|(\phi)}{B} \leq \frac{\eta}{4 K}
$$

Then using (7.26) and (7.29) we may select $s^{\prime}<t$ so that

$$
\left|[s, t]-E_{B}\right|<\frac{(t-s) \eta}{2 K} \text { if } s^{\prime}<s<t,
$$

and

$$
\delta\left(V_{r}^{\gamma}, \phi\right)\left(h\left(V_{r}^{\gamma}, \cdot\right)\right) \leq \delta\left(V_{t}^{\gamma}, \phi\right)\left(h\left(V_{t}^{\gamma}, \cdot\right)\right)+\frac{\eta}{2} \text { if } s^{\prime}<r<t, r \in E_{B} .
$$

Thus (7.25) imply that if $s^{\prime}<s<t$, then

$$
\begin{aligned}
\left\|V_{t}^{\gamma}\right\|(\phi)-\left\|V_{s}^{\gamma}\right\|(\phi) \leq & \int_{s}^{t} \delta\left(V_{r}^{\gamma}, \phi\right)\left(h\left(V_{r}^{\gamma}, \cdot\right)\right) d r \\
\leq & \int_{[t, s] \cap E_{B}} \delta\left(V_{t}^{\gamma}, \phi\right)\left(h\left(V_{t}^{\gamma}, \cdot\right)\right)+\frac{\eta}{2} d r \\
& +\int_{[t, s]-E_{B}} K d r \text { by }(7.24),(7.31) .
\end{aligned}
$$

Utilizing (7.30) we obtain

$$
D^{-}\left\|V_{t}^{\gamma}\right\|(\phi) \leq \delta\left(V_{t}^{\gamma}, \phi\right)\left(h\left(V_{t}^{\gamma}, \cdot\right)\right)+\eta
$$

for each $\eta>0$. Consequently, (7.22) implies

$$
D^{-}\left\|V^{\gamma}\right\|(\phi) \leq \delta\left(V^{\gamma}, \phi\right)\left(h\left(V^{\gamma}, \cdot\right)\right)
$$

for each $\phi \in C_{c}^{1}\left(\mathbb{R}^{n}\right), \phi \geq 0$.

\section{Suppose now}

$$
D^{+}\left\|V_{t}^{\gamma}\right\|(\phi)>-\infty
$$


$D^{+}$denoting the upper right derivative. In particular then,

$$
\lim _{s \rightarrow r^{+}}\left\|V_{s}^{\gamma}\right\|(\phi)=\left\|V_{t}^{\gamma}\right\|(\phi)
$$

Now, similarly to step 2 , we have

$$
\limsup _{s \rightarrow t^{+}} \frac{\left|[t, s]-E_{B}\right|}{s-t} \leq \frac{1}{B}\left[K-D^{+}\left\|V_{t}^{\gamma}\right\|(\phi)\right]
$$

Suppose $t_{k} \in(t, \infty) \cap E_{B}(k=1,2, \ldots), t_{k} \rightarrow t^{+}$. As in step 3 we have the bounds

$$
\sup _{k}\left\|\delta V_{t_{k}}^{\gamma}\right\|\left(W_{\eta}\right),\left\|V_{t_{k}}^{\gamma}\right\|\left(W_{\eta}\right)<\infty
$$

for $\eta>0, W_{\eta}$ as before. Hence Allard's Compactness Theorem for Integral Varifolds implies $\left\{V_{t_{k}}^{\gamma}\left\llcorner W_{\eta}\right\}_{k=1}^{\infty}\right.$ is precompact in $I V_{n-1}\left(\mathbb{R}^{n}\right)$. Consequently, passing as necessary to a subsequence and reindexing, we may assume

$$
V_{t_{k}}^{\gamma}\left\llcornerW _ { \eta } \rightarrow U _ { t } \left\llcorner W_{\eta}\right.\right.
$$

for some $U_{t} \in I V_{n-1}\left(\mathbb{R}^{n}\right)$. In particular,

$$
\left\|V_{t_{k}}^{\gamma}\right\|\left\llcornerW _ { \eta } \rightarrow \| U _ { t } \| \left\llcorner W_{\eta}\right.\right.
$$

Passing to a further subsequence, we may in fact show

$$
\left\|V_{t_{k}}^{\gamma}\right\|_{\llcorner} W_{\eta_{j}} \rightarrow\left\|U_{t}\right\|\left\llcorner W_{\eta_{j}}\right.
$$

for some sequence $\eta_{j} \rightarrow 0$, and in particular

$$
\left\|V_{t_{k}}^{\gamma}\right\|(\phi) \rightarrow\left\|U_{t}\right\|(\phi)
$$

But then, in view of (7.34),

$$
\left\|V_{t}^{\gamma}\right\|(\phi)=\left\|U_{t}\right\|(\phi) .
$$

However, according to (7.14)-(7.18), we have $\left\|U_{t}\right\| \leq\left\|V_{t}^{\gamma}\right\|$ as measures. Thus (7.36) forces $\left\|V_{t}\right\|\left\llcorner\{\phi>0\}=\left\|U_{t}\right\|_{\llcorner}\{\phi>0\}\right.$. Hence

$$
V_{r}^{\gamma}\llcorner\{\phi>0\} \text { is an integral varifold. }
$$

We now mimic step 4 above to show

$$
D^{+}\left\|V_{t}^{\gamma}\right\|(\phi) \leq \delta\left(V_{t}^{\gamma}, \phi\right)\left(h\left(V_{t}^{\gamma}, \cdot\right)\right)
$$

provided (7.33) obtains. 


\section{References}

[1] Allard, W. On the first variation of a varifold. Ann. of Math. 95, 417-491 (1972).

[2] Brakke, K. The Motion of a Surface by Its Mean Curvature. Princeton University Press, Princeton, NJ, 1978.

[3] Chen, Y.-G., Giga, Y., and Goto, S. Uniqueness and existence of viscosity solutions of generalized mean curvature flow equations. J. Diff. Geom. 33 (1991)

[4] Chen, Y.-G., Giga, Y., and Goto, S. Analysis towards snow crystal growth, preprint, 1991.

[5] Dacorogna, B. Weak Continuity and Weak Lower Semicontinuity of Nonlinear Functionals. Springer-Verlag, Berlin, 1982.

[6] Evans, L. C. Weak Convergence Methods for Nonlinear Partial Differential Equations. CBMS Lecture Series \#74. American Mathematical Society, Providence, RI, 1990.

[7] Evans, L. C., Soner, M., and Souganidis, P. E. Phase transitions and generalized motion by mean curvature. Comm. Pure Appl. Math., to appear.

18] Evans, L. C., and Spruck, J. Motion of level sets by mean curvature, I. J. Diff. Geom. 33, 635-681 (1991).

[9] Evans, L. C., and Spruck, J. Motion of level sets by mean curvature, II. Trans. AMS 330, 321-332 (1992).

[10] Evans, L. C., and Spruck, J. Motion of level sets by mean curvature, III. J. Geom. Anal. 2, 121-150 (1992).

[11] Federer, H. Geometric Measure Theory. Springer-Verlag, Berlin, 1969.

[12] Giga, Y., Goto, S., and Ishii, H. Global existence of weak solutions for interface equations coupled with diffusion equations, preprint, 1991.

[13] Giga, Y., Goto, S., Ishii, H., and Sato, M.-H. Comparison principle and convexity preserving properties for singular degenerate parabolic equations on unbounded domains. Indian Univ. Math. J., to appear.

[14] Huisken, G. Flow by mean curvature of convex surfaces into spheres. J. Diff. Geom. 20, 237-266 (1984).

[15] Ilmanen, T. Generalized flow of sets by mean curvature on a manifold, preprint, 1990.

[16] Ilmanen, T. Motion of level sets and varifolds by mean curvature. Ph.D. thesis, University of California, Berkeley, 1991

[17] Ohta, T., Jasnow, D., and Kawasaki, K. Universal scaling in the motion of random interfaces. Phys. Rev. Lett. 49, 1223-1226 (1982).

[18] Osher, S., and Sethian, J. Fronts propagating with curvature dependent speed: algorithms based on Hamilton-Jacobi formulations. J. Comp. Phys. 79, 12-49 (1988).

[19] Sethian, J. Curvature and evolution of fronts. Comm. Math. Phys. 101, 487-499 (1985).

[20] Sethian, J. Recent numerical algorithms for hypersurfaces moving with curvature dependent speed: Hamilton-Jacobi equations and conservation laws. J. Diff. Geom. 31, 131-161 (1990).

[21] Simon, L. Lectures on Geometric Measure Theory. Proceedings of the Centre for Mathematical Analysis \#3, Australian National University, Canberra, 1984.

[22] Soner, M. Motion of a set by the curvature of its boundary, preprint, 1990.

[23] Tartar, L. Compensated compactness and applications to partial differential equations. In Heriot-Watt Symposium, Vol. IV. Pitman Press, New York, 1979.

Received June 9, 1992

Department of Mathematics, University of California, Berkeley, CA 94720 Department of Mathematics, University of Massachusetts, Amherst, MA 01003 